\title{
Uniform bound and convergence for elliptic homogenization problems
}

\author{
Li-Ming Yeh $^{1}$
}

Received: 23 May 2015 / Accepted: 28 August 2015 / Published online: 8 September 2015

(C) Fondazione Annali di Matematica Pura ed Applicata and Springer-Verlag Berlin Heidelberg 2015

\begin{abstract}
Uniform bound and convergence for the solutions of elliptic homogenization problems are concerned. The problem domain has a periodic microstructure; it consists of a connected subregion with high permeability and a disconnected matrix block subset with low permeability. Let $\epsilon \in(0,1)$ denote the size ratio of the period to the whole domain, and let $\omega^{2} \in(0,1)$ denote the permeability ratio of the disconnected matrix block subset to the connected subregion. For elliptic equations with diffusion depending on the permeability, the elliptic solutions are smooth in the connected subregion but change rapidly in the disconnected matrix block subset. More precisely, the solutions in the connected subregion can be bounded uniformly in $\omega, \epsilon$ in Hölder norm, but not in the matrix block subset. It is known that the elliptic solutions converge to a solution of some homogenized elliptic equation as $\omega, \epsilon$ converge to 0 . In this work, the $L^{p}$ convergence rate for $p \in(2, \infty]$ is derived. Depending on strongly coupled or weakly coupled case, the convergence rate is related to the factors $\omega, \epsilon, \frac{\omega}{\epsilon}$ for the former and related to the factors $\omega, \epsilon$ for the latter.
\end{abstract}

Keywords Elliptic homogenization problem $\cdot$ Permeability $\cdot$ Two-phase media

Mathematics Subject Classification 35J05 $\cdot 35 \mathrm{~J} 15 \cdot 35 \mathrm{~J} 25$

\section{Introduction}

Uniform bound and convergence for the solutions of elliptic homogenization problems are presented. The problems have applications in contaminant transport in the subsurface, heat transfer in two-phase media, the stress in composite materials, and so on (see $[3,10,17,18]$ ). The problem domain $\Omega \subset \mathbb{R}^{n}(n=2,3)$ contains two subsets, a periodic connected subregion with high permeability and a periodic disconnected matrix block subset with low permeability.

Li-Ming Yeh

liming@math.nctu.edu.tw

1 Department of Applied Mathematics, National Chiao Tung University,

Hsinchu 30050, Taiwan, ROC 
Let $\epsilon \in(0,1)$ be a parameter, $Y \equiv(0,1)^{n}$ consist of a subdomain $Y_{m}$ completely surrounded by another connected subdomain $Y_{f}\left(\equiv Y \backslash \overline{Y_{m}}\right), \Omega(\epsilon) \equiv\{x \in \Omega \mid \operatorname{dist}(x, \partial \Omega)>\epsilon\}, \Omega_{m}^{\epsilon} \equiv$ $\left\{x \mid x \in \epsilon\left(Y_{m}+j\right) \subset \Omega(\epsilon)\right.$ for some $\left.j \in \mathbb{Z}^{n}\right\}$ be the disconnected matrix block subset of $\Omega$, $\Omega_{f}^{\epsilon} \equiv \Omega \backslash \overline{\Omega_{m}^{\epsilon}}$ be the connected subregion of $\Omega$, and $\partial \Omega$ (resp. $\partial \Omega_{m}^{\epsilon}$ ) be the boundary of $\Omega$ (resp. $\Omega_{m}^{\epsilon}$ ). The problem that we consider is

$$
\begin{cases}-\nabla \cdot\left(\mathbf{E}_{\omega^{2}, \epsilon} \mathbf{K}_{\epsilon} \nabla \Psi_{\omega, \epsilon}+V_{\omega, \epsilon}\right)+\mathbf{T}_{\omega, \epsilon} \Psi_{\omega, \epsilon}=G_{\omega, \epsilon} & \text { in } \Omega \\ \Psi_{\omega, \epsilon}=0 & \text { on } \partial \Omega\end{cases}
$$

where $\omega, \epsilon \in(0,1), \mathbf{E}_{v, \epsilon} \equiv\left\{\begin{array}{ll}1 & \text { in } \Omega_{f}^{\epsilon} \\ v & \text { in } \Omega_{m}^{\epsilon}\end{array}\right.$ for any $v>0, \mathbf{K}_{\epsilon}(x) \equiv \mathbf{K}\left(\frac{x}{\epsilon}\right), \mathbf{K}$ is a positive periodic function in $\mathbb{R}^{n}$ with period $Y, \mathbf{T}_{\omega, \epsilon}$ is a nonnegative function, and $V_{\omega, \epsilon}, G_{\omega, \epsilon}$ are given functions. It is known that if $\mathbf{K}_{\epsilon}, \mathbf{T}_{\omega, \epsilon}, V_{\omega, \epsilon}, G_{\omega, \epsilon}$ are smooth in $\Omega_{f}^{\epsilon} \cup \Omega_{m}^{\epsilon}$, a piecewise smooth solution of (1.1) exists uniquely [19]. The $H^{1}$ norm of the solution in the high-permeability subregion $\Omega_{f}^{\epsilon}$ is bounded uniformly in $\omega, \epsilon$ when $V_{\omega, \epsilon}, G_{\omega, \epsilon}$ are small in $\Omega_{m}^{\epsilon}$. However, that may not be the case for the solution in the low-permeability subset $\Omega_{m}^{\epsilon}$ (see Remark 2.2). Also the second-order derivatives of the solution of (1.1) may not be bounded uniformly in $\omega, \epsilon$ in the high-permeability subregion $\Omega_{f}^{\epsilon}$ even when $V_{\omega, \epsilon}, G_{\omega, \epsilon}$ are bounded uniformly in $\omega, \epsilon$ and are small in $\Omega_{m}^{\epsilon}$ (see Remark 3.1). By homogenization theory (see [7,17,23]), if $\omega, \epsilon$ become small, the solution of (1.1) approaches to a solution of some homogenized elliptic differential equation. So it seems that, if both $\omega, \epsilon$ are small, the solution of the homogenized elliptic differential equation is a good approximation of the solution of (1.1). We shall see in Sect. 2 that the solution of (1.1) can be approximated by the solution of the homogenized elliptic differential equation plus some functions, which are the solutions of mutually independent local problems.

Lipschitz estimate and $W^{2, p}$ estimate for the solutions of the uniform elliptic equations with discontinuous coefficients had been considered in [20,21]. For the uniform elliptic case of (1.1) (that is, $\omega=1$ ), uniform bound and convergence results were also studied. For example, uniform Hölder, $W^{1, p}$, and Lipschitz estimates in $\epsilon$ for uniform elliptic case of (1.1) with Hölder coefficients were proved in [4,5]. Uniform $W^{1, p}$ estimate in $\epsilon$ for uniform elliptic case of (1.1) with continuous coefficients was shown in [13], and the same problem with VMO coefficients could be found in [25]. Uniform Lipschitz estimate in $\epsilon$ for the Laplace equation in periodic perforated domains was studied in [24]. By [7,17,23], the solution of uniform elliptic case of (1.1) with Dirichlet boundary condition converges to a solution of some homogenized elliptic equation with convergence rate $\epsilon$ in $L^{2}$ norm and with convergence rate $\epsilon^{1 / 2}$ in $H^{1}$ norm as $\epsilon$ closes to 0 .

In this work, we consider the non-uniform elliptic case of (1.1) with discontinuous coefficients. We derive uniform Hölder estimates in $\omega, \epsilon$ for the solution of (1.1) as well as derive $L^{p}$ convergence estimates for $p \in(2, \infty]$ for the approximation of the solution of (1.1).

One interesting related problem is the study of the equations with contrasting diffusivity in a fibered medium, that is, a conductivity medium reinforced by an $\epsilon$-periodic lattice of highly or lowly conducting thin rods (see $[6,8,9,11,12,26]$ and references therein). In [9], homogenization problem of degenerate Poisson equations in a fiber-reinforced structure was considered. In [12], the article tried to find a suitable conductivity medium which corresponding the prescribed Dirichlet problem with the non-local term. In [26], the authors analyzed nonlinear monotone conduction problems in a fibered medium. A two-scale convergence result to a non-local homogenized equation was shown. In [8], weak convergence of the solution of a $p$-Laplacian-type equation in a fiber-reinforced structure was shown. In [6], a 
spectral problem of a Poisson equations in a bounded domain with a high contrast in both stiffness and density was studied. In [11], the article considered the uniform regularity of the elliptic solutions in a fibered medium with $\omega=\omega(\epsilon) \gg 1$ and $\epsilon \ll 1$. Uniform $W^{1,6}$ bound in $\epsilon$ and uniform $C^{1, v}$ convergence estimate in $\epsilon$ of the solutions were derived in an interior region with a distance $\epsilon^{\tau}$ away from the highly conducting thin rods for some $\nu, \tau>0$ (the distance constraint is required). Different from [11], we derive uniform Hölder estimates in $\omega, \epsilon$ for the solution of (1.1) in the high-permeability region $\Omega_{f}^{\epsilon}$ (the distance constraint is not required). Moreover, we obtain the uniform convergence estimates in $\omega, \epsilon$ for the solution of (1.1) in the whole domain $\Omega$.

The rest of the work is organized as follows: Notation and main results are stated in Sect. 2. In Sect. 3, we derive a priori uniform estimates for interface problems. Uniform Hölder estimates for the non-uniform elliptic solutions in heterogeneous media are considered in Sect. 4. $L^{p}$ convergence estimates for elliptic homogenization problems are presented in Sect. 5.

\section{Notation and main result}

If $D \subset \mathbb{R}^{n}$ is a set, $\bar{D}$ denotes the closure of the set $D, \mathcal{X}_{D}$ is the characteristic function on $D,|D|$ is the volume of $D, \partial D$ is the boundary of $D$, and $D / r=\{x \mid r x \in D\}$ for $r>0$. $B_{r}(x)$ denotes a ball centered at $x$ with radius $r>0$. If $\mathbf{B}_{1}, \mathbf{B}_{2}$ are Banach spaces, $\left\|\varphi_{1}, \cdots, \varphi_{m}\right\|_{\mathbf{B}_{1}} \equiv\left\|\varphi_{1}\right\|_{\mathbf{B}_{1}}+\cdots+\left\|\varphi_{m}\right\|_{\mathbf{B}_{1}}$ and $\|\varphi\|_{\mathbf{B}_{1} \cap \mathbf{B}_{2}} \equiv\|\varphi\|_{\mathbf{B}_{1}}+\|\varphi\|_{\mathbf{B}_{2}} \cdot C^{k, \alpha}$ denotes the Hölder space with norm $\|\cdot\|_{C^{k, \alpha}} ; W^{s, p}$ denotes the Sobolev space with norm $\|\cdot\|_{W^{s, p}}$; $[\varphi]_{C^{0, \alpha}}$ is the Hölder semi-norm of $\varphi ; L^{p}(D) \equiv W^{0, p}(D) ; H^{s}(D) \equiv W^{s, 2}(D)$ for $k \geq 0$, $\alpha \in(0,1], s \geq-1, p \in[1, \infty]$ (see [2,16]). $C_{0}^{\infty}(D)$ is the space of infinitely differentiable functions with support in $D ; C_{\text {per }}^{\infty}\left(\mathbb{R}^{n}\right)$ is the space of infinitely differentiable $Y$-periodic functions in $\mathbb{R}^{n} ; W_{\text {per }}^{s, p}(D)$ is the closure of $C_{\text {per }}^{\infty}\left(\mathbb{R}^{n}\right)$ under the $W^{s, p}$ norm for $s \geq 0, p \in$ $[1, \infty] ; H_{p e r}^{1}(D) \equiv W_{\text {per }}^{1,2}(D) ; L_{p e r}^{\infty}(D) \equiv W_{\text {per }}^{0, \infty}(D)$. For $p \geq 2, W_{0}^{1, p}(D) \equiv\{\varphi \in$ $W^{1, p}(D) \mid \varphi=0$ on $\left.\partial D\right\}$ and $H_{0}^{1}(D)=W_{0}^{1,2}(D)$. For any $\varphi \in L^{1}(D)$ and $r>0$,

$$
(\varphi)_{x, r} \equiv f_{D \cap B_{r}(x)} \varphi(y) \mathrm{d} y \equiv \frac{1}{\left|D \cap B_{r}(x)\right|} \int_{D \cap B_{r}(x)} \varphi(y) \mathrm{d} y .
$$

If $\overrightarrow{\mathbf{n}}$ is an outward normal vector on $\partial Y_{m}$, we define, for any function $\varphi$ and $x \in \partial Y_{m}$,

$$
\varphi_{, \pm}(x) \equiv \lim _{t \rightarrow 0^{+}} \varphi(x \pm t \overrightarrow{\mathbf{n}}), \quad\lfloor\varphi\rfloor(x)=\varphi_{,+}(x)-\varphi_{,-}(x)
$$

Similarly, if $\overrightarrow{\mathbf{n}}^{\epsilon}$ is an outward normal vector on $\partial \Omega_{m}^{\epsilon}$, we define, for any $x \in \partial \Omega_{m}^{\epsilon}$,

$$
\varphi_{, \pm}(x) \equiv \lim _{t \rightarrow 0^{+}} \varphi\left(x \pm t \overrightarrow{\mathbf{n}}^{\epsilon}\right), \quad\lfloor\varphi\rfloor(x)=\varphi_{,+}(x)-\varphi,-(x)
$$

Next we recall an extension result in [1].

Remark 2.1 For any $\epsilon \in(0,1)$ and $p \in[1, \infty)$, there are a constant $\ell_{1}\left(Y_{f}, p\right)$ and a linear continuous extension operator $\Pi_{\epsilon}: W^{1, p}\left(\Omega_{f}^{\epsilon}\right) \rightarrow W^{1, p}(\Omega)$ such that 
(1) if $\varphi \in W^{1, p}\left(\Omega_{f}^{\epsilon}\right)$, then

$$
\left\{\begin{array}{l}
\Pi_{\epsilon} \varphi=\varphi \text { in } \Omega_{f}^{\epsilon} \text { almost everywhere, } \\
\left\|\Pi_{\epsilon} \varphi\right\|_{L^{p}(\Omega)} \leq \ell_{1}\|\varphi\|_{L^{p}\left(\Omega_{f}^{\epsilon}\right)}, \\
\left\|\nabla \Pi_{\epsilon} \varphi\right\|_{L^{p}(\Omega)} \leq \ell_{1}\|\nabla \varphi\|_{L^{p}\left(\Omega_{f}^{\epsilon}\right)}, \\
\ell_{2} \leq \Pi_{\epsilon} \leq \ell_{3} \quad \text { if } \varphi \in L^{\infty}\left(\Omega_{f}^{\epsilon}\right) \text { and } \ell_{2} \leq \varphi \leq \ell_{3}, \\
\Pi_{\epsilon} \varphi=\zeta \text { in } \Omega \text { if } \varphi=\left.\zeta\right|_{\Omega_{f}^{\epsilon}} \text { for some linear function } \zeta \text { in } \Omega,
\end{array}\right.
$$

(2) if $r>0, \epsilon / r<1$, and $\zeta(x) \equiv \varphi(r x)$, then $\Pi_{\epsilon / r} \zeta(x)=\left(\Pi_{\epsilon} \varphi\right)(r x)$.

If $\varphi \in W^{1, p}(\Omega)$ for any $p \geq 1$, then $\left.\Pi_{\epsilon} \varphi\right|_{\Omega_{f}^{\epsilon}} \in W^{1, p}(\Omega)$ denotes the extension function of

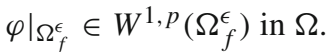

We briefly state our main results. Theorems 2.1-2.2 are uniform bound estimates for the solution of (1.1). Theorem 2.1 is for the strongly coupled case (i.e., $0<\mathbf{M}_{0} \leq \mathbf{T}_{\omega, \epsilon} \leq \mathbf{M}$ in $\Omega$ ), and Theorem 2.2 is for the weakly coupled case (i.e., $0 \leq \mathbf{E}_{1 / \omega, \epsilon} \mathbf{T}_{\omega, \epsilon} \leq \mathbf{M}$ in $\Omega$ ). Proofs of Theorems 2.1-2.2 are given in Sect. 4. Theorems 2.3-2.5 are convergence estimates for the solution of (1.1). Theorem 2.3 is for the strongly coupled case, and Theorems 2.4-2.5 are for the weakly coupled case. Proofs of Theorems 2.3-2.5 are given in Sect. 5.

\section{Theorem 2.1 Suppose}

A1. $\Omega \subset \mathbb{R}^{n}$ is a $C^{2,1}$ domain for $n \in\{2,3\}, Y_{m}$ is a smooth simply connected subdomain of $Y, \overline{Y_{m}} \subset Y$,

A2. $\mathbf{K} \in H_{\text {per }}^{1}\left(\mathbb{R}^{n}\right)$ is a positive function, $\|\nabla \mathbf{K}\|_{L^{\infty}(Y)}$ is small compared with $\min _{x \in Y} \mathbf{K}(x)$, and $\mathbf{K} \in C^{1, \alpha}\left(\overline{Y_{f}}\right) \cap C^{1, \alpha}\left(\overline{Y_{m}}\right)$ for some $\alpha \in(0,1)$,

A3. $\omega, \epsilon \in(0,1), \delta \in(0,3), V_{\omega, \epsilon}, G_{\omega, \epsilon} \in L^{n+\delta}(\Omega)$,

A4. $\mathbf{M}_{0}, \mathbf{M}>0, \mathbf{T}_{\omega, \epsilon}(x) \in\left[\mathbf{M}_{0}, \mathbf{M}\right]$ for all $x \in \Omega$,

then a $H^{1}(\Omega)$ solution of $(1.1)$ exists uniquely and there is a constant $\omega_{0} \in(0,1)$ (depending on $\left.\delta, \mathbf{K}, \mathbf{M}, Y_{f}, \Omega\right)$ such that, for $\omega<\omega_{0}$ and $\frac{\omega}{\epsilon}>\ell_{4}>0$,

$$
\left[\Psi_{\omega, \epsilon}\right]_{C^{0, \mu}\left(\overline{\Omega_{f}^{\epsilon}}\right)}+\sup _{\substack{j \in \mathbb{Z}^{n} \\ \epsilon\left(Y_{m}+j\right) \subset \Omega_{m}^{\epsilon}}} \omega\left[\Psi_{\omega, \epsilon}\right]_{C^{0, \mu}\left(\epsilon\left(\overline{Y_{m}}+j\right)\right)} \leq c\left\|\mathbf{E}_{1 / \omega, \epsilon} V_{\omega, \epsilon}, G_{\omega, \epsilon}\right\|_{L^{n+\delta}(\Omega)},
$$

where $\ell_{4}$ is any number, $\mu \equiv \frac{\delta}{n+\delta}$, and $c$ is a positive constant independent of $\omega, \epsilon$.

Theorem 2.2 Besides A1-A3, if

A4'. $\mathbf{E}_{1 / \omega, \epsilon} \mathbf{T}_{\omega, \epsilon}(x) \in[0, \mathbf{M}]$ for all $x \in \Omega$,

then a $H^{1}(\Omega)$ solution of $(1.1)$ exists uniquely and there is a constant $\omega_{0} \in(0,1)$ (depending on $\left.\delta, \mathbf{K}, \mathbf{M}, Y_{f}, \Omega\right)$ such that, for any $\omega<\omega_{0}$,

$$
\begin{gathered}
{\left[\Psi_{\omega, \epsilon}\right]_{C^{0, \mu}\left(\overline{\Omega_{f}^{\epsilon}}\right)}+\sup _{\substack{j \in \mathbb{Z}^{n} \\
\epsilon\left(Y_{m}+j\right) \subset \Omega_{m}^{\epsilon}}} \omega^{\lambda}\left[\Psi_{\omega, \epsilon}\right]_{C^{0, \mu}\left(\epsilon\left(\overline{Y_{m}}+j\right)\right)}} \\
\leq c\left(\left\|\mathbf{E}_{1 / \omega, \epsilon} V_{\omega, \epsilon}, \mathbf{E}_{\max \{1, \epsilon / \omega\}} G_{\omega, \epsilon}\right\|_{L^{n+\delta}(\Omega)},\right.
\end{gathered}
$$

where $\mu \equiv \frac{\delta}{n+\delta}$ and $c$ is independent of $\omega, \epsilon . \operatorname{In}(2.4), \lambda=\left\{\begin{array}{ll}\frac{3}{2} & \text { if } \mathbf{T}_{\omega, \epsilon} \neq 0 \\ 1 & \text { if } \mathbf{T}_{\omega, \epsilon}=0\end{array}\right.$. 
From Theorem 2.1 and Theorem 2.2, we know that if the right-hand side of (2.3) or (2.4) is bounded, the Hölder norm of the solution of (1.1) in the connected high-permeability region $\Omega_{f}^{\epsilon}$ is bounded uniformly in $\omega, \epsilon$, but the solution in $\Omega_{m}^{\epsilon}$ may change rapidly when $\omega, \epsilon$ are small. This is different from uniform elliptic equation case, where the solution is bounded uniformly in the whole domain. To obtain the uniform Hölder estimate for the solution of (1.1), the condition $\frac{\omega}{\epsilon} \geq \ell_{4}>0$ is needed in strongly coupled case but not in weakly coupled case. Below is one example to show that the Hölder norm and the $H^{1}$ norm of the solution of (1.1) in $\Omega_{m}^{\epsilon}$ in general are not be bounded uniformly in $\omega, \epsilon$.

Remark 2.2 Suppose $\varphi \in C_{\text {per }}^{\infty}\left(\mathbb{R}^{n}\right)$ and $\varphi$ in the cell $Y \equiv(0,1)^{n}$ has support in $Y_{m}$, we define, for any $\epsilon \in(0,1)$,

$$
\Psi_{\omega, \epsilon}(x) \equiv \begin{cases}0 & \text { if } x \in \Omega_{f}^{\epsilon} \\ \varphi\left(\frac{x}{\epsilon}\right) & \text { if } x \in \Omega_{m}^{\epsilon}\end{cases}
$$

Then $\Psi_{\omega, \epsilon}$ satisfies

$$
\begin{cases}-\nabla \cdot\left(\mathbf{E}_{\omega^{2}, \epsilon} \nabla \Psi_{\omega, \epsilon}\right)=G_{\omega, \epsilon} & \text { in } \Omega \\ \Psi_{\omega, \epsilon}=0 & \text { on } \partial \Omega\end{cases}
$$

where $G_{\omega, \epsilon}(x)=-\omega^{2} \epsilon^{-2} \Delta \varphi\left(\frac{x}{\epsilon}\right) \mathcal{X}_{\Omega_{m}^{\epsilon}}$. Note $\left[\Psi_{\omega, \epsilon}\right]_{C^{0, \mu}\left(\epsilon\left(\overline{Y_{m}}+j\right)\right)}=\epsilon^{-\mu}[\varphi]_{C^{0, \mu}\left(\overline{Y_{m}}+j\right)}$, $\left\|\nabla \Psi_{\omega, \epsilon}\right\|_{L^{2}\left(\Omega_{m}^{\epsilon}\right)} \approx \epsilon^{-1}\|\nabla \varphi\|_{L^{2}\left(Y_{m}\right)}$, and $\left\|G_{\omega, \epsilon}\right\|_{L^{n+\delta}\left(\Omega_{m}^{\epsilon}\right)} \approx \omega^{2} \epsilon^{-2}\|\Delta \varphi\|_{L^{n+\delta}\left(Y_{m}\right)}$ where $\delta>0, \mu \equiv \frac{\delta}{n+\delta}$. Here $A \approx B$ means that $A$ is almost like $B$ times a constant when $\epsilon$ is small. If $\omega \leq \epsilon<1$, then the right-hand side of (2.4) is finite and (2.4) holds for $\Psi_{\omega, \epsilon}$. But the $C^{0, \mu}$ norm and the $H^{1}$ norm of $\Psi_{\omega, \epsilon}$ in $\Omega_{m}^{\epsilon}$ are not bounded uniformly in $\epsilon \in(0,1)$.

Next we state convergence results. Set $\mathbb{A}_{m} \equiv\left\{x \in \mathbb{R}^{n} \mid x \in \cup_{j \in \mathbb{Z}^{n}}\left(Y_{m}+j\right)\right\}, \mathbb{A}_{f} \equiv$ $\mathbb{R}^{n} \backslash \overline{\mathbb{A}_{m}}$, and $\mathbb{E}_{v} \equiv\left\{\begin{array}{ll}1 & \text { in } Y_{f} \\ v & \text { in } Y_{m}\end{array}\right.$ for any $v>0$. We find $\mathbb{X}_{v}^{(i)} \in H_{p e r}^{1}\left(\mathbb{R}^{n}\right)$ for $v \in(0,1]$ and $i \in\{1,2, \cdots, n\}$ satisfying

$$
\left\{\begin{array}{l}
-\nabla \cdot\left(\mathbb{E}_{v^{2}} \mathbf{K}\left(\nabla \mathbb{X}_{v}^{(i)}+\vec{e}_{i}\right)\right)=0 \quad \text { in } Y, \\
\int_{Y_{f}} \mathbb{X}_{v}^{(i)}(y) \mathrm{d} y=0,
\end{array}\right.
$$

find $\mathbb{X}_{0}^{(i)} \in H_{p e r}^{1}\left(\mathbb{A}_{f}\right) \cap H_{p e r}^{1}\left(\mathbb{A}_{m}\right)$ for $i \in\{1,2, \cdots, n\}$ satisfying

$$
\begin{cases}-\nabla \cdot\left(\mathbf{K}\left(\nabla \mathbb{X}_{0}^{(i)}+\vec{e}_{i}\right)\right)=0 & \text { in } Y_{f}, \\ \mathbb{X}_{0}^{(i)}=0 & \text { in } Y_{m}, \\ \mathbf{K}\left(\nabla \mathbb{X}_{0}^{(i)}+\vec{e}_{i}\right)_{,+} \cdot \overrightarrow{\mathbf{n}}=0 & \text { on } \partial Y_{m} \\ \int_{Y_{f}} \mathbb{X}_{0}^{(i)}(y) \mathrm{d} y=0, & \end{cases}
$$


and find $\mathbb{W}_{\beta} \in H_{\text {per }}^{1}\left(\mathbb{A}_{f}\right) \cap H_{\text {per }}^{1}\left(\mathbb{A}_{m}\right)$ for $\beta>0, \mathbf{T} \in L_{\text {per }}^{\infty}\left(\mathbb{R}^{n}\right)$, and $\mathbf{T} \geq 0$ satisfying

$$
\begin{aligned}
& \begin{cases}\beta^{2} \nabla \cdot\left(\mathbf{K} \nabla \mathbb{W}_{\beta}\right)-\mathbf{T} \mathbb{W}_{\beta}=0 & \text { in } Y_{m}, \\
\mathbb{W}_{\beta}=1 & \text { on } \partial Y_{m},\end{cases} \\
& \begin{cases}\nabla \cdot\left(\mathbf{K} \nabla \mathbb{W}_{\beta}\right)-\mathbf{T}=\frac{-1}{\left|Y_{f}\right|}\left(\int_{Y_{f}} \mathbf{T} \mathrm{d} y+\int_{Y_{m}} \mathbf{T} \mathbb{W}_{\beta} \mathrm{d} y\right) & \text { in } Y_{f}, \\
\left\lfloor\mathbb{E}_{\beta^{2}} \mathbf{K} \nabla \mathbb{W}_{\beta}\right\rfloor \cdot \overrightarrow{\mathbf{n}}=0 & \text { on } \partial Y_{m}, \\
\int_{Y_{f}} \mathbb{W}_{\beta}(y) \mathrm{d} y=0, & \end{cases}
\end{aligned}
$$

where $\vec{e}_{i}$ is a unit vector in the $i$ th coordinate direction, $\left|Y_{f}\right|$ is the volume of $Y_{f}$, and $\overrightarrow{\mathbf{n}}$ is an outward normal vector on $\partial Y_{m}$. See (2.1) for (2.6) 3 and (2.8) 2 . Let $\mathbb{X}_{v, s}^{(i)}(x) \equiv s \mathbb{X}_{v}^{(i)}\left(\frac{x}{s}\right)$ $\mathbb{X}_{\nu, s} \equiv\left(\mathbb{X}_{\nu, s}^{(1)}, \cdots, \mathbb{X}_{\nu, s}^{(n)}\right)$, and $\mathbb{W}_{\beta, s, i}(x) \equiv s^{i} \mathbb{W}_{\beta}\left(\frac{x}{s}\right)$ for any $v \in[0,1], s \in(0,1), \beta>0$, $i \in \mathbb{Z}$. By Lax-Milgram Theorem [16], (2.5)-(2.8) are uniquely solvable. Denote by $\Xi_{v}$ for $v \in[0,1]$ a $n \times n$ matrix function whose $(i, j)$-component is $\partial_{i} \mathbb{X}_{v}^{(j)}$. By remark in pages 17-19, 94-95 [17],

$$
\mathcal{K}_{v} \equiv \int_{Y_{f} \cup Y_{m}} \mathbb{E}_{v^{2}} \mathbf{K}\left(I+\Xi_{v}(y)\right) \mathrm{d} y \text { for } v \in[0,1]
$$

is a constant symmetric positive definite matrix. Here $I$ is the identity matrix.

If, in addition to A1-A4,

A5. $\mathbf{T}_{\omega, \epsilon}(x)=\mathbf{T}\left(\frac{x}{\epsilon}\right)>0$ and $\mathbf{T} \in C_{p e r}^{0, \alpha}\left(\mathbb{R}^{n}\right)$ for some $\alpha>0$,

A6. $\left\|\mathbf{E}_{1 / \omega, \epsilon} G_{\omega, \epsilon}\right\|_{L^{n+\delta}(\Omega)}+\left\|G_{\omega, \epsilon}\right\|_{W^{1, n+\delta}\left(\Omega_{f}^{\epsilon}\right)}$ is bounded independent of $\omega, \epsilon$,

the solution of (1.1) with $V_{\omega, \epsilon}=0$ satisfies $\left\|\mathbf{E}_{\omega, \epsilon} \nabla \Psi_{\omega, \epsilon}, \Psi_{\omega, \epsilon}\right\|_{L^{2}(\Omega)} \leq c$ (independent of $\omega, \epsilon)$. Suppose $\omega, \epsilon \rightarrow 0$ and $\frac{\omega}{\epsilon} \rightarrow \sigma \in[0, \infty]$, by tracing the proof of Theorem 2.3 [3], we can extract a subsequence (same notation for subsequence) such that

$$
\left\{\begin{array}{l}
\mathbf{E}_{\omega^{2}, \epsilon} \mathbf{K}_{\epsilon} \nabla \Psi_{\omega, \epsilon} \rightarrow \mathcal{K}_{0} \nabla \Psi \\
\mathbf{T}_{\omega, \epsilon} \Psi_{\omega, \epsilon} \rightarrow\left|Y_{f}\right| \mathcal{T}_{\sigma} \Psi \quad \text { in } L^{2}(\Omega) \text { weakly }, \\
G_{\omega, \epsilon} \rightarrow\left|Y_{f}\right| \mathcal{G}
\end{array}\right.
$$

where $\mathcal{K}_{0}$ is defined in (2.9) with $v=0,\left|Y_{f}\right|$ is the volume of $Y_{f}$, and

$$
\mathcal{T}_{\sigma}= \begin{cases}\frac{1}{\left|Y_{f}\right|} \int_{Y} \mathbf{T d} y & \text { if } \sigma=\infty, \\ \frac{1}{\left|Y_{f}\right|}\left(\int_{Y_{f}} \mathbf{T} \mathrm{d} y+\int_{Y_{m}} \mathbf{T} \mathbb{W}_{\sigma} \mathrm{d} y\right) & \text { if } \sigma \in(0, \infty), \\ \frac{1}{\left|Y_{f}\right|} \int_{Y_{f}} \mathbf{T} \mathrm{d} y & \text { if } \sigma=0 .\end{cases}
$$

See (2.7) for $\mathbb{W}_{\sigma}$. The $\Psi$ in (2.10) satisfies

$$
\begin{cases}-\nabla \cdot\left(\mathcal{K}_{0} \nabla \Psi\right)+\left|Y_{f}\right| \mathcal{T}_{\sigma} \Psi=\left|Y_{f}\right| \mathcal{G} & \text { in } \Omega, \\ \Psi=0 & \text { on } \partial \Omega .\end{cases}
$$


By Theorem 9.19 [16] and A6,

$$
\|\Psi\|_{W^{3, n+\delta}(\Omega)} \leq c\|\mathcal{G}\|_{W^{1, n+\delta}(\Omega)},
$$

where $c$ is a constant depending on $\mathcal{K}_{0}, \mathbf{M},\left|Y_{f}\right|, \Omega$. Now for any $\omega, \epsilon \in(0,1)$ and on any $\epsilon\left(Y_{m}+j\right) \subset \Omega_{m}^{\epsilon}$ for some $j \in \mathbb{Z}^{n}$, we consider

$$
\begin{cases}-\nabla \cdot\left(\omega^{2} \mathbf{K}_{\epsilon} \nabla \phi_{\omega, \epsilon}^{(j)}\right)+\mathbf{T}_{\omega, \epsilon} \phi_{\omega, \epsilon}^{(j)}=G_{\omega, \epsilon} & \text { in } \epsilon\left(Y_{m}+j\right), \\ \phi_{\omega, \epsilon}^{(j)}=\Psi & \text { on } \epsilon\left(\partial Y_{m}+j\right),\end{cases}
$$

where $\Psi$ is the solution of (2.12). By Lax-Milgram Theorem [16], A5-A6, and (2.13), the $\phi_{\omega, \epsilon}^{(j)}$ of (2.14) is solvable uniquely in $H^{1}\left(\epsilon\left(Y_{m}+j\right)\right)$. By Theorem 8.24 and Theorem 8.29 [16], $\phi_{\omega, \epsilon}^{(j)} \in L^{\infty}\left(\epsilon\left(Y_{m}+j\right)\right)$. Moreover,

Theorem 2.3 Suppose Al-A6 and $V_{\omega, \epsilon}=0$ in (1.1). There is a constant $\omega_{0} \in(0,1)$ such that, for any $\omega \in\left(0, \omega_{0}\right)$ and $\epsilon \in(0,1)$,

1. if $\omega, \epsilon \rightarrow 0$ and $\frac{\omega}{\epsilon} \rightarrow \infty$, the solutions of (1.1), (2.12), and (2.14) satisfy

$$
\begin{gathered}
\left\|\mathbf{E}_{\omega, \epsilon}\left(\Psi_{\omega, \epsilon}-\Psi\right)\right\|_{L^{\infty}(\Omega)}+\left\|\Psi_{\omega, \epsilon}-\sum_{\substack{j \in \mathbb{Z}^{n} \\
\epsilon\left(Y_{m}+j\right) \subset \Omega_{m}^{\epsilon}}} \phi_{\omega, \epsilon}^{(j)}\right\|_{L^{\infty}\left(\Omega_{m}^{\epsilon}\right)} \\
\leq c\left(\left\|G_{\omega, \epsilon}-\mathcal{G}\right\|_{L^{n+\delta}\left(\Omega_{f}^{\epsilon}\right)}+\max \{\omega, \epsilon / \omega\}\right),
\end{gathered}
$$

2. if $\omega, \epsilon \rightarrow 0$ and $\frac{\omega}{\epsilon} \rightarrow \sigma \in(0, \infty)$, the solutions of (1.1), (2.12), and (2.14) satisfy

$$
\begin{gathered}
\| \mathbf{E}_{\omega, \epsilon}\left(\Psi_{\omega, \epsilon}-\left(\mathcal{X}_{\Omega_{f}^{\epsilon}}+\mathbb{W} \frac{\omega}{\epsilon}, \epsilon, 0\right.\right. \\
\left.\left.\mathcal{X}_{\Omega_{m}^{\epsilon}}\right) \Psi\right)\left\|_{L^{\infty}(\Omega)}+\right\| \Psi_{\omega, \epsilon}-\sum_{\substack{j \in \mathbb{Z}^{n} \\
\epsilon\left(Y_{m}+j\right) \subset \Omega_{m}^{\epsilon}}} \phi_{\omega, \epsilon}^{(j)} \|_{L^{\infty}\left(\Omega_{m}^{\epsilon}\right)} \\
\leq c\left(\left\|G_{\omega, \epsilon}-\mathcal{G}\right\|_{L^{n+\delta}\left(\Omega_{f}^{\epsilon}\right)}+\max \left\{\omega, \epsilon,\left|\omega^{2} /(\sigma \epsilon)^{2}-1\right|\right\}\right),
\end{gathered}
$$

3. if $\omega, \epsilon \rightarrow 0$ and $\frac{\omega}{\epsilon} \rightarrow 0$, the solutions of (1.1) and (2.12) satisfy

$$
\begin{aligned}
& \left\|\Psi_{\omega, \epsilon}-\left(\mathcal{X}_{\Omega_{f}^{\epsilon}}+\mathbb{W}_{\frac{\omega}{\epsilon}, \epsilon, 0} \mathcal{X}_{\Omega_{m}^{\epsilon}}\right) \Psi\right\|_{L^{n+\delta}(\Omega)} \\
& \quad \leq c\left(\left\|G_{\omega, \epsilon}-\mathcal{G}\right\|_{L^{n+\delta}\left(\Omega_{f}^{\epsilon}\right)}+\max \left\{\omega, \epsilon,\left|\omega \epsilon^{-1} \ln \left(\omega \epsilon^{-1}\right)\right|^{\frac{1}{2}}\right\}\right),
\end{aligned}
$$

where $c$ is a constant independent of $\omega, \epsilon$.

Theorem 2.3, based on Theorem 2.1, is a convergence result for (1.1) in strongly coupled case. Note that $L^{\infty}$ convergence estimate is obtained for $\frac{\omega}{\epsilon} \rightarrow \sigma>0$ case and that only $L^{n+\delta}$ convergence estimate is available for $\frac{\omega}{\epsilon} \rightarrow 0$ case. Next we present convergence estimates for the solutions of (1.1) in weakly coupled case (that is, Theorems 2.4 and 2.5).

Besides A1-A3, A4', and A6, if

$$
\text { A7. } \mathbf{E}_{1 / \omega, \epsilon} \mathbf{T}_{\omega, \epsilon}(x)=\mathbf{P}\left(\frac{x}{\epsilon}\right) \geq 0 \text { and } \mathbf{P} \in L_{p e r}^{\infty}\left(\mathbb{R}^{n}\right) \cap C^{0, \alpha}\left(\overline{\mathbb{A}_{f}}\right) \text { for some } \alpha>0 \text {, }
$$

the solution of (1.1) with $V_{\omega, \epsilon}=0$ satisfies $\left\|\mathbf{E}_{\omega, \epsilon} \nabla \Psi_{\omega, \epsilon}, \mathbf{T}_{\omega, \epsilon}^{1 / 2} \Psi_{\omega, \epsilon}\right\|_{L^{2}(\Omega)} \leq c$ (independent of $\omega, \epsilon)$. By compactness principle [3,17],

$$
\left\{\begin{array}{l}
\mathbf{E}_{\omega^{2}, \epsilon} \mathbf{K}_{\epsilon} \nabla \Psi_{\omega, \epsilon} \rightarrow \mathcal{K}_{0} \nabla \Psi \\
\mathbf{T}_{\omega, \epsilon} \Psi_{\omega, \epsilon} \rightarrow\left|Y_{f}\right| \breve{\mathcal{T}} \Psi \\
G_{\omega, \epsilon} \rightarrow\left|Y_{f}\right| \mathcal{G}
\end{array} \quad \text { in } L^{2}(\Omega) \text { weakly as } \omega, \epsilon \rightarrow 0\right.
$$


where $\breve{\mathcal{T}}\left(=\frac{1}{\left|Y_{f}\right|} \int_{Y_{f}} \mathbf{P}(y) \mathrm{d} y\right)$ is a constant vector and $\mathcal{K}_{0}$ is defined in (2.9) with $v=0$. Similar to (2.12)-(2.13), the $\Psi$ in (2.15) satisfies

$$
\begin{cases}-\nabla \cdot\left(\mathcal{K}_{0} \nabla \Psi\right)+\left|Y_{f}\right| \breve{\mathcal{T}} \Psi=\left|Y_{f}\right| \mathcal{G} & \text { in } \Omega, \\ \Psi=0 & \text { on } \partial \Omega, \\ \|\Psi\|_{W^{3, n+\delta}(\Omega)} \leq c\|\mathcal{G}\|_{W^{1, n+\delta}(\Omega)}, & \end{cases}
$$

where $\left|Y_{f}\right|$ is the volume of $Y_{f}$ and $c$ is a constant depending on $\mathcal{K}_{0}, \mathbf{M},\left|Y_{f}\right|, \Omega$.

We have the following result:

Theorem 2.4 Assume A1-A3, A4', A6-A7, and $V_{\omega, \epsilon}=0$ in (1.1). There is a $\omega_{0} \in(0,1)$ such that if $\omega<\omega_{0}$, then the solutions of (1.1), (2.16), and (2.14) with $\Psi$ obtained from (2.16) satisfy

$$
\begin{gathered}
\left\|\mathbf{E}_{\omega^{3 / 2}, \epsilon} \Psi_{\omega, \epsilon}-\Psi\right\|_{L^{\infty}(\Omega)}+\left\|\Psi_{\omega, \epsilon}-\sum_{\substack{j \in \mathbb{Z}^{n} \\
\epsilon\left(Y_{m}+j\right) \subset \Omega_{m}^{\epsilon}}} \phi_{\omega, \epsilon}^{(j)}\right\|_{L^{\infty}\left(\Omega_{m}^{\epsilon}\right)} \\
\leq c\left(\left\|G_{\omega, \epsilon}-\mathcal{G}\right\|_{L^{n+\delta}\left(\Omega_{f}^{\epsilon}\right)}+\max \{\omega, \epsilon\}\right),
\end{gathered}
$$

where $c$ is a constant independent of $\omega, \epsilon$. See (2.16) for $\mathcal{G}$.

Under A1-A3 and A6, the solution of (1.1) with $V_{\omega, \epsilon}=\mathbf{T}_{\omega, \epsilon}=0$ satisfies $\left\|\mathbf{E}_{\omega, \epsilon} \nabla \Psi_{\omega, \epsilon}\right\|_{L^{2}(\Omega)}$ $\leq c$ (independent of $\omega, \epsilon$ ). By compactness principle [3,17],

$$
\left\{\begin{array}{l}
\mathbf{E}_{\omega^{2}, \epsilon} \mathbf{K}_{\epsilon} \nabla \Psi_{\omega, \epsilon} \rightarrow \mathcal{K}_{0} \nabla \Psi \\
G_{\omega, \epsilon} \rightarrow\left|Y_{f}\right| \mathcal{G}
\end{array} \quad \text { in } L^{2}(\Omega) \text { weakly as } \omega, \epsilon \rightarrow 0,\right.
$$

where $\mathcal{K}_{0}$ is defined in (2.9) with $v=0$. The $\Psi$ in (2.17) satisfies

$$
\begin{cases}-\nabla \cdot\left(\mathcal{K}_{0} \nabla \Psi\right)=\left|Y_{f}\right| \mathcal{G} & \text { in } \Omega, \\ \Psi=0 & \text { on } \partial \Omega, \\ \|\Psi\|_{W^{3, n+\delta}(\Omega)} \leq c\|\mathcal{G}\|_{W^{1, n+\delta}(\Omega)}, & \end{cases}
$$

where $\left|Y_{f}\right|$ is the volume of $Y_{f}$ and $c$ is a constant depending on $\mathcal{K}_{0},\left|Y_{f}\right|, \Omega$. We also have

Theorem 2.5 Assume A1-A3, A6, and $V_{\omega, \epsilon}=\mathbf{T}_{\omega, \epsilon}=0$ in (1.1). There is a $\omega_{0} \in(0,1)$ such that if $\omega<\omega_{0}$, the solutions of (1.1), (2.18), and (2.14) with $\mathbf{T}_{\omega, \epsilon}=0$ and $\Psi$ from (2.18) satisfy

$$
\begin{gathered}
\left\|\mathbf{E}_{\omega, \epsilon} \Psi_{\omega, \epsilon}-\Psi\right\|_{L^{\infty}(\Omega)}+\left\|\Psi_{\omega, \epsilon}-\sum_{\substack{j \in \mathbb{Z}^{n} \\
\epsilon\left(Y_{m}+j\right) \subset \Omega_{m}^{\epsilon}}} \phi_{\omega, \epsilon}^{(j)}\right\|_{L^{\infty}\left(\Omega_{m}^{\epsilon}\right)} \\
\leq c\left(\left\|G_{\omega, \epsilon}-\mathcal{G}\right\|_{L^{n+\delta}\left(\Omega_{f}^{\epsilon}\right)}+\max \{\omega, \epsilon\}\right),
\end{gathered}
$$

where $c$ is a constant independent of $\omega, \epsilon$. See (2.17) for $\mathcal{G}$.

Theorems 2.3, 2.4, 2.5 imply if $\omega, \epsilon,\left\|G_{\omega, \epsilon}-\mathcal{G}\right\|_{L^{n+\delta}\left(\Omega_{f}^{\epsilon}\right)}$ are small enough, the homogenized solution $\Psi$ of (2.12) or (2.16) or (2.18) is a good approximation of the solution of (1.1) in the connected subregion $\Omega_{f}^{\epsilon}$, but the $\Psi$ may not be a good approximation of the solution of (1.1) in the disconnected subset $\Omega_{m}^{\epsilon}$. In the disconnected subset $\Omega_{m}^{\epsilon}$, the solution of (1.1) can be approximated by the solution of (2.14). One also notes that $\sum_{\substack{\epsilon\left(Y_{m}+j\right) \subset \Omega_{m}^{\epsilon} \\ j \in \mathbb{Z}^{n}}} \phi_{\omega, \epsilon}^{(j)}$ is obtained by solving mutually independent local problems. 


\section{A priori uniform estimates for interface problems}

Let $\Gamma(x-y)$ denote the fundamental solution of the Laplace equation in $\mathbb{R}^{n}$; see $\S 6.2$ [14]. Define a single-layer and a double-layer potentials as, for any smooth function $\varphi$ on the boundary $\partial Y_{m}$ of $Y_{m}$,

$$
\left\{\begin{array}{l}
\mathcal{S}_{\partial Y_{m}}(\varphi)(x) \equiv \int_{\partial Y_{m}} \Gamma(x-y) \varphi(y) \mathrm{d} y \\
\mathcal{L}_{\partial Y_{m}}(\varphi)(x) \equiv \int_{\partial Y_{m}} \nabla_{y} \Gamma(x-y) \overrightarrow{\mathbf{n}}_{y} \varphi(y) \mathrm{d} y
\end{array} \quad \text { for } x \in \partial Y_{m},\right.
$$

where $\overrightarrow{\mathbf{n}}_{y}$ is the unit vector outward normal to $\partial Y_{m}$. By tracing the argument of Lemma 4.1 [27], we know

Lemma 3.1 For any $p \in(1, \infty), i \in\{0,1\}$, and $\alpha \in(0,1)$, the linear operators

$$
\left\{\begin{array}{l}
\mathcal{S}_{\partial Y_{m}}: W^{i-\frac{1}{p}, p}\left(\partial Y_{m}\right) \rightarrow W^{i+1-\frac{1}{p}, p}\left(\partial Y_{m}\right) \\
\mathcal{L}_{\partial Y_{m}}: W^{i+1-\frac{1}{p}, p}\left(\partial Y_{m}\right) \rightarrow W^{i+2-\frac{1}{p}, p}\left(\partial Y_{m}\right) \\
\mathcal{S}_{\partial Y_{m}}: C^{1, \alpha}\left(\partial Y_{m}\right) \rightarrow C^{2, \alpha}\left(\partial Y_{m}\right) \\
\mathcal{L}_{\partial Y_{m}}: C^{1, \alpha}\left(\partial Y_{m}\right) \rightarrow C^{2, \alpha}\left(\partial Y_{m}\right)
\end{array}\right.
$$

are bounded; the operator $I-\ell \mathcal{L}_{\partial Y_{m}}$ is continuously invertible in $W^{i+1-\frac{1}{p}, p}\left(\partial Y_{m}\right)$ and in $C^{2, \alpha}\left(\partial Y_{m}\right)$ for $\ell \in[-2,2]$; there is a constant $c$ independent of $\ell$ so that

$$
\begin{cases}\|\varphi\|_{W^{i+1-\frac{1}{p}, p}\left(\partial Y_{m}\right)} \leq c\left\|\left(I-\ell \mathcal{L}_{\partial Y_{m}}\right)(\varphi)\right\|_{W^{i+1-\frac{1}{p}, p}\left(\partial Y_{m}\right)} & \text { for } \varphi \in W^{i+1-\frac{1}{p}, p}\left(\partial Y_{m}\right), \\ \|\varphi\|_{C^{2, \alpha}\left(\partial Y_{m}\right)} \leq c\left\|\left(I-\ell \mathcal{L}_{\partial Y_{m}}\right)(\varphi)\right\|_{C^{2, \alpha}\left(\partial Y_{m}\right)} & \text { for } \varphi \in C^{2, \alpha}\left(\partial Y_{m}\right),\end{cases}
$$

where I is the identity operator.

By A1, let us assume

$$
\left\{\begin{array}{l}
Y_{m} \subset \mathbf{D}_{1} \subset \mathbf{D}_{2} \subset Y, \\
\min \left\{\operatorname{dist}\left(Y_{m}, \partial \mathbf{D}_{1}\right), \operatorname{dist}\left(\mathbf{D}_{1}, \partial \mathbf{D}_{2}\right), \operatorname{dist}\left(\mathbf{D}_{2}, \partial Y\right)\right\}>0 .
\end{array}\right.
$$

Lemma 3.2 Under $A 1-A 2, \omega \in(0,1], p \in(n, 6), \mathbf{M}>0$, and $\mathbb{P}_{\omega}(x) \in[0, \mathbf{M}]$ for all $x \in Y$, any solution of

$$
-\nabla \cdot\left(\mathbb{E}_{\omega^{2}} \mathbf{K} \nabla U_{\omega}+Q_{\omega}\right)+\mathbb{E}_{\omega} \mathbb{P}_{\omega} U_{\omega}=F_{\omega} \quad \text { in } Y
$$

satisfies

$$
\begin{aligned}
& \left\|\mathbb{E}_{\omega^{3 / 2}} U_{\omega}\right\|_{W^{1, p}\left(\mathbf{D}_{1} \backslash \overline{Y_{m}}\right) \cap W^{1, p}\left(Y_{m}\right)} \leq c\left(\left\|U_{\omega}\right\|_{L^{2}\left(Y_{f}\right)}+\left\|\mathbb{E}_{1 / \omega} Q_{\omega}\right\|_{L^{2}(Y)}\right) \\
& \left.\quad+\left\|\mathbb{E}_{1 / \omega} F_{\omega}\right\|_{H^{-1}(Y)}+\left\|\mathbb{E}_{1 / \sqrt{\omega}} Q_{\omega}\right\|_{L^{p}(Y)}+\left\|\mathbb{E}_{1 / \sqrt{\omega}} F_{\omega}\right\|_{W^{-1, p}(Y)}\right),
\end{aligned}
$$

where $c$ is a constant independent of $\omega$. See Sect. 2 for $\mathbb{E}_{v}$.

Proof Let $p \in(n, 6)$ and $c$ denote a constant independent of $\omega$.

Step 1: Assume $Q_{\omega} \in W_{0}^{1, p}\left(Y_{f}\right) \cap W_{0}^{1, p}\left(Y_{m}\right), F_{\omega} \in L^{p}(Y)$, and consider

$$
\begin{cases}-\nabla \cdot\left(\mathbb{E}_{\omega^{2}} \mathbf{K} \nabla \varphi_{\omega}+Q_{\omega}\right)+\mathbb{E}_{\omega} \mathbb{P}_{\omega} \varphi_{\omega}=F_{\omega} & \text { in } \mathbf{D}_{2}, \\ \varphi_{\omega}=0 & \text { on } \partial \mathbf{D}_{2} .\end{cases}
$$


The unique existence of a solution of (3.4) in $H^{1}\left(\mathbf{D}_{2}\right)$ is known by Lax-Milgram Theorem [16]. By Theorem 7.26 and Poincaré inequality [16],

$$
\begin{gathered}
\left\|\varphi_{\omega}\right\|_{L^{p}\left(\mathbf{D}_{2} \backslash \overline{Y_{m}}\right)}+\omega^{1 / 2}\left\|\mathbb{P}_{\omega}^{1 / 2} \varphi_{\omega}\right\|_{W^{-1, p}\left(Y_{m}\right)} \leq c\left\|\mathbb{E}_{\omega} \nabla \varphi_{\omega}, \mathbb{E}_{\omega^{1 / 2}} \mathbb{P}_{\omega}^{1 / 2} \varphi_{\omega}\right\|_{L^{2}\left(\mathbf{D}_{2}\right)} \\
\leq c\left(\left\|\mathbb{E}_{1 / \omega} Q_{\omega}\right\|_{L^{2}\left(\mathbf{D}_{2}\right)}+\left\|\mathbb{E}_{1 / \omega} F_{\omega}\right\|_{H^{-1}\left(\mathbf{D}_{2}\right)}\right. \\
\left.\quad+\left\|\mathbb{E}_{1 / \sqrt{\omega}} Q_{\omega}\right\|_{L^{p}\left(\mathbf{D}_{2}\right)}+\left\|\mathbb{E}_{1 / \sqrt{\omega}} F_{\omega}\right\|_{W^{-1, p}\left(\mathbf{D}_{2}\right)}\right) \equiv c \mathcal{I}_{\omega} .
\end{gathered}
$$

By (3.4)-(3.5) and [22], we have

$$
\left\|\varphi_{\omega}\right\|_{W^{1, p}\left(\mathbf{D}_{2} \backslash \mathbf{D}_{1}\right)} \leq c \mathcal{I}_{\omega} .
$$

See (3.1) for $\mathbf{D}_{1}$. Let $\widehat{\varphi}$ in $Y_{m}$ be the solution of

$$
\left\{\begin{array}{l}
-\nabla \cdot\left(\omega^{2} \widehat{\mathbf{k}} \nabla \widehat{\varphi}+\omega^{2}(\mathbf{K}-\widehat{\mathbf{k}}) \nabla \varphi_{\omega}+Q_{\omega}\right)=F_{\omega}-\omega \mathbb{P}_{\omega} \varphi_{\omega} \quad \text { in } Y_{m}, \\
\left.\widehat{\varphi}\right|_{\partial Y_{m}}=0
\end{array}\right.
$$

and $\widehat{\varphi}$ in $\mathbf{D}_{2} \backslash \overline{Y_{m}}$ be the solution of

$$
\begin{cases}-\nabla \cdot\left(\widehat{\mathbf{K}} \nabla \widehat{\varphi}+(\mathbf{K}-\widehat{\mathbf{K}}) \nabla \varphi_{\omega}+Q_{\omega}\right)=F_{\omega}-\mathbb{P}_{\omega} \varphi_{\omega} & \text { in } \mathbf{D}_{2} \backslash \overline{Y_{m}} \\ \left.\widehat{\varphi}\right|_{\partial\left(\mathbf{D}_{2} \backslash \overline{Y_{m}}\right)}=0 & \end{cases}
$$

where $\widehat{\mathbf{K}}, \widehat{\mathbf{k}}$ are two constants in the interval $\left(\min _{Y} \mathbf{K}, \max _{Y} \mathbf{K}\right)$. By [22] and (3.5),

$$
\left\{\begin{array}{l}
\omega^{2} \widehat{\mathbf{k}}\|\widehat{\varphi}\|_{W^{1, p}\left(Y_{m}\right)} \leq c\left(\omega^{1 / 2} \mathcal{I}_{\omega}+\omega^{2}\left\|(\mathbf{K}-\widehat{\mathbf{k}}) \nabla \varphi_{\omega}\right\|_{L^{p}\left(Y_{m}\right)}\right) \\
\widehat{\mathbf{K}}\|\widehat{\varphi}\|_{W^{1, p}\left(\mathbf{D}_{2} \backslash \overline{Y_{m}}\right)} \leq c\left(\mathcal{I}_{\omega}+\left\|(\mathbf{K}-\widehat{\mathbf{K}}) \nabla \varphi_{\omega}\right\|_{L^{p}\left(\mathbf{D}_{2} \backslash \overline{Y_{m}}\right)}\right)
\end{array}\right.
$$

If we define $\check{\varphi} \equiv \varphi_{\omega}-\widehat{\varphi}$ in $\mathbf{D}_{2}$, then (3.4) and (3.7)-(3.8) imply

$$
\begin{cases}\Delta \check{\varphi}=0 & \text { in } \mathbf{D}_{2} \backslash \partial Y_{m}, \\ \lfloor\check{\varphi}\rfloor=0 & \text { on } \partial Y_{m}, \\ \lfloor\check{\mathbb{E}} \nabla \check{\varphi}\rfloor \cdot \overrightarrow{\mathbf{n}}_{y}=\mathcal{F} / \widehat{\mathbf{K}} & \text { on } \partial Y_{m}, \\ \check{\varphi}=0 & \text { on } \partial \mathbf{D}_{2},\end{cases}
$$

where $\check{\mathbb{E}} \equiv\left\{\begin{array}{ll}\omega^{2} \widehat{\mathbf{k}} / \widehat{\mathbf{K}} & \text { in } Y_{m} \\ 1 & \text { in } Y_{f}\end{array}\right.$ and $\overrightarrow{\mathbf{n}}_{y}$ is the unit vector outward normal to $\partial Y_{m}$. See (2.1) for $(3.10)_{2,3}$. Since $Q_{\omega} \in W_{0}^{1, p}\left(Y_{f}\right) \cap W_{0}^{1, p}\left(Y_{m}\right)$,

$$
\left.\mathcal{F} \equiv\left(\omega^{2} \widehat{\mathbf{k}} \nabla \widehat{\varphi},--\widehat{\mathbf{K}} \nabla \widehat{\varphi}_{,+}+\omega^{2}(\mathbf{K}-\widehat{\mathbf{k}}) \nabla \varphi_{\omega,-}-(\mathbf{K}-\widehat{\mathbf{K}}) \nabla \varphi_{\omega,+}\right) \cdot \overrightarrow{\mathbf{n}}_{y}\right|_{\partial Y_{m}} .
$$

By (3.9),

$$
\begin{gathered}
\|\mathcal{F}\|_{W^{-\frac{1}{p}, p}\left(\partial Y_{m}\right)} \leq c\left(\mathcal{I}_{\omega}+\omega^{2}\|\mathbf{K}-\widehat{\mathbf{k}}\|_{L^{\infty}\left(Y_{m}\right)}\left\|\varphi_{\omega}\right\|_{W^{1, p}\left(Y_{m}\right)}\right. \\
\left.+\|\mathbf{K}-\widehat{\mathbf{K}}\|_{L^{\infty}\left(\mathbf{D}_{2} \backslash \overline{Y_{m}}\right)}\left\|\varphi_{\omega}\right\|_{W^{1, p}\left(\mathbf{D}_{2} \backslash \overline{Y_{m}}\right)}\right) .
\end{gathered}
$$

By Green's formula, (3.10), and Theorem 6.5.1 [14], we see that

$$
\left\{\begin{array}{l}
\check{\varphi} / 2+\mathcal{L}_{\partial Y_{m}}(\check{\varphi})=\mathcal{S}_{\partial Y_{m}}\left(\left.\partial_{\mathbf{n}_{y}} \check{\varphi}_{,-}\right|_{\partial Y_{m}}\right) \\
\check{\varphi} / 2-\mathcal{L}_{\partial Y_{m}}(\check{\varphi})=-\mathcal{S}_{\partial Y_{m}}\left(\partial_{\mathbf{n}_{y}} \check{\varphi},+\left.\right|_{\partial Y_{m}}\right)+\mathcal{S}_{\partial \mathbf{D}_{2}}\left(\left.\partial_{\mathbf{n}_{y}} \check{\varphi}\right|_{\partial \mathbf{D}_{2}}\right)
\end{array} \quad \text { on } \partial Y_{m},\right.
$$


where $\left.\partial_{\mathbf{n}_{y}} \check{\varphi}\right|_{\partial \mathbf{D}_{2}}$ is the normal derivative of $\check{\varphi}$ on $\partial \mathbf{D}_{2}$. So we have

$$
\left(I-\frac{2(1-\check{\omega})}{1+\check{\omega}} \mathcal{L}_{\partial Y_{m}}\right) \check{\varphi}=\frac{2 \mathcal{S}_{\partial \mathbf{D}_{2}}\left(\left.\partial_{\mathbf{n}_{y}} \check{\varphi}\right|_{\partial \mathbf{D}_{2}}\right)}{1+\check{\omega}}-\frac{2 \mathcal{S}_{\partial Y_{m}}(\mathcal{F})}{(1+\check{\omega}) \widehat{\mathbf{K}}} \quad \text { on } \partial Y_{m},
$$

where $\check{\omega} \equiv \omega^{2} \widehat{\mathbf{k}} / \widehat{\mathbf{K}}$. Then (3.6), (3.9), (3.12), and Lemma 3.1 imply

$$
\left\{\begin{array}{l}
\|\check{\varphi}\|_{W^{1-\frac{1}{p}, p}\left(\partial Y_{m}\right)} \leq c\left(\|\mathcal{F}\|_{W^{-\frac{1}{p}, p}\left(\partial Y_{m}\right)}+\left\|\partial_{\mathbf{n}_{y}} \check{\varphi}\right\|_{W^{-\frac{1}{p}, p}\left(\partial \mathbf{D}_{2}\right)}\right), \\
\left\|\partial_{\mathbf{n}_{y}} \check{\varphi}\right\|_{W^{-\frac{1}{p}, p}\left(\partial \mathbf{D}_{2}\right)} \leq c\left(\mathcal{I}_{\omega}+\left\|(\mathbf{K}-\widehat{\mathbf{K}}) \nabla \varphi_{\omega}\right\|_{L^{p}\left(\mathbf{D}_{2} \backslash \overline{Y_{m}}\right)}\right) .
\end{array}\right.
$$

(3.10)-(3.11) and (3.13) imply

$$
\begin{aligned}
& \|\check{\varphi}\|_{W^{1, p}\left(\mathbf{D}_{2} \backslash \overline{Y_{m}}\right) \cap W^{1, p}\left(Y_{m}\right)} \leq c\left(\mathcal{I}_{\omega}+\omega^{2}\|\mathbf{K}-\widehat{\mathbf{k}}\|_{L^{\infty}\left(Y_{m}\right)}\left\|\varphi_{\omega}\right\|_{W^{1, p}\left(Y_{m}\right)}\right. \\
& \left.\quad+\|\mathbf{K}-\widehat{\mathbf{K}}\|_{L^{\infty}\left(\mathbf{D}_{2} \backslash \overline{Y_{m}}\right)}\left\|\varphi_{\omega}\right\|_{W^{1, p}\left(\mathbf{D}_{2} \backslash \overline{Y_{m}}\right)}\right) .
\end{aligned}
$$

Together with (3.9), we obtain

$$
\begin{gathered}
\left\|\mathbb{E}_{\omega^{3 / 2}} \varphi_{\omega}\right\|_{W^{1, p}\left(\mathbf{D}_{2} \backslash \overline{Y_{m}}\right) \cap W^{1, p}\left(Y_{m}\right)} \leq c\left(\mathcal{I}_{\omega}+\omega^{3 / 2}\|\mathbf{K}-\widehat{\mathbf{k}}\|_{L^{\infty}\left(Y_{m}\right)}\left\|\varphi_{\omega}\right\|_{W^{1, p}\left(Y_{m}\right)}\right. \\
\left.+\|\mathbf{K}-\widehat{\mathbf{K}}\|_{L^{\infty}\left(\mathbf{D}_{2} \backslash \overline{Y_{m}}\right)}\left\|\varphi_{\omega}\right\|_{W^{1, p}\left(\mathbf{D}_{2} \backslash \overline{Y_{m}}\right)}\right)
\end{gathered}
$$

By A2, we obtain

$$
\left\|\mathbb{E}_{\omega^{3 / 2}} \varphi_{\omega}\right\|_{W^{1, p}\left(\mathbf{D}_{2} \backslash \overline{Y_{m}}\right) \cap W^{1, p}\left(Y_{m}\right)} \leq c \mathcal{I}_{\omega} .
$$

Step 2: Note $W_{0}^{1, p}\left(Y_{f}\right)$ (resp. $\left.W_{0}^{1, p}\left(Y_{m}\right)\right)$ is dense in $L^{p}\left(Y_{f}\right)$ (resp. $L^{p}\left(Y_{m}\right)$ ) as well as $L^{p}(Y)$ is dense in $W^{-1, p}(Y)$. By a limiting argument, we see that if $Q_{\omega} \in L^{p}(Y)$ and $F_{\omega} \in W^{-1, p}(Y)$, the solution of (3.4) still satisfies (3.14).

Step 3: Let $\eta$ be a smooth function satisfying $\eta \in C_{0}^{\infty}\left(\mathbf{D}_{2}\right), \eta \in[0,1], \eta=1$ in $\mathbf{D}_{1}$, $\|\nabla \eta\|_{W^{1, \infty}\left(\mathbf{D}_{2}\right)} \leq c$. Multiply (3.2) by $\eta$ to obtain

$$
\left\{\begin{aligned}
-\nabla & \cdot\left(\mathbb{E}_{\omega^{2}} \mathbf{K} \nabla\left(U_{\omega} \eta\right)-\mathbf{K} U_{\omega} \nabla \eta+Q_{\omega} \eta\right)+\mathbb{E}_{\omega} \mathbb{P}_{\omega} U_{\omega} \eta & & \\
& =F_{\omega} \eta-\left(\mathbf{K} \nabla U_{\omega}+Q_{\omega}\right) \nabla \eta & & \text { in } \mathbf{D}_{2}, \\
U_{\omega} \eta & =0 & & \text { on } \partial \mathbf{D}_{2} .
\end{aligned}\right.
$$

By the result of Step 2, we have

$$
\begin{gathered}
\left\|\mathbb{E}_{\omega^{3 / 2}} U_{\omega}\right\|_{W^{1, p}\left(\mathbf{D}_{1} \backslash \overline{Y_{m}}\right) \cap W^{1, p}\left(Y_{m}\right)} \leq c\left(\left\|U_{\omega}\right\|_{L^{p}\left(\mathbf{D}_{2} \backslash \mathbf{D}_{1}\right)}+\left\|\mathbb{E}_{1 / \sqrt{\omega}} Q_{\omega}\right\|_{L^{p}(Y)}\right. \\
\left.+\left\|\mathbb{E}_{1 / \sqrt{\omega}} F_{\omega}\right\|_{W^{-1, p}(Y)}+\left\|\mathbb{E}_{1 / \omega} Q_{\omega}\right\|_{L^{2}(Y)}+\left\|\mathbb{E}_{1 / \omega} F_{\omega}\right\|_{H^{-1}(Y)}\right) .
\end{gathered}
$$

Let $\tilde{\eta}$ be another smooth function satisfying $\tilde{\eta} \in C_{0}^{\infty}(Y), \tilde{\eta} \in[0,1], \tilde{\eta}=1$ in $\mathbf{D}_{2}$, $\|\nabla \tilde{\eta}\|_{W^{1, \infty}(Y)} \leq c$. Multiply (3.2) by $\tilde{\eta}$ and then use energy method to get

$$
\left\|U_{\omega}\right\|_{L^{p}\left(\mathbf{D}_{2} \backslash \mathbf{D}_{1}\right)} \leq c\left(\left\|U_{\omega}\right\|_{L^{2}\left(Y_{f}\right)}+\left\|\mathbb{E}_{1 / \omega} Q_{\omega}\right\|_{L^{2}(Y)}+\left\|\mathbb{E}_{1 / \omega} F_{\omega}\right\|_{H^{-1}(Y)}\right) .
$$

Together with (3.15), we obtain (3.3). 
Modifying the argument for Lemma 3.2 and employing Lemma 3.1, we see

Lemma 3.3 Under $A 1-A 2, \omega \in(0,1]$, and $p \in(n, \infty)$, any solution of

$$
-\nabla \cdot\left(\mathbb{E}_{\omega^{2}} \mathbf{K} \nabla U_{\omega}+Q_{\omega}\right)=F_{\omega} \quad \text { in } Y
$$

satisfies

$$
\begin{aligned}
& \left\|\mathbb{E}_{\omega^{i}} U_{\omega}\right\|_{W^{1, p}\left(\mathbf{D}_{1} \backslash \overline{Y_{m}}\right) \cap W^{1, p}\left(Y_{m}\right)} \\
& \quad \leq c\left(\left\|U_{\omega}\right\|_{L^{2}\left(Y_{f}\right)}+\left\|\mathbb{E}_{\omega^{i-2}} Q_{\omega}\right\|_{L^{p}(Y)}+\left\|\mathbb{E}_{\omega^{i-2}} F_{\omega}\right\|_{W^{-1, p}(Y)}\right), \\
& \left\|U_{\omega}\right\|_{W^{2, p}\left(\mathbf{D}_{1} \backslash \overline{Y_{m}}\right) \cap W^{2, p}\left(Y_{m}\right)} \\
& \quad \leq c\left(\left\|U_{\omega}\right\|_{L^{2}\left(Y_{f}\right)}+\left\|\mathbb{E}_{\omega^{-2}} Q_{\omega}\right\|_{W^{1, p}\left(Y_{f}\right) \cap W^{1, p}\left(Y_{m}\right)}+\left\|\mathbb{E}_{\omega^{-2}} F_{\omega}\right\|_{L^{p}(Y)}\right), \\
& \left\|\mathbb{E}_{\omega^{i}} U_{\omega}\right\|_{C^{2, \alpha}\left(\overline{\mathbf{D}_{1} \backslash Y_{m}}\right) \cap C^{2, \alpha}\left(\overline{Y_{m}}\right)} \leq c\left(\left\|U_{\omega}\right\|_{L^{2}\left(Y_{f}\right)}+\left\|\mathbb{E}_{\omega^{i-2}} Q_{\omega}\right\|_{C^{1, \alpha}\left(\overline{Y_{f}}\right) \cap C^{1, \alpha}\left(\overline{Y_{m}}\right)}\right. \\
& \left.\quad+\left\|\mathbb{E}_{\omega^{i-2}} F_{\omega}\right\|_{C^{0, \alpha}\left(\overline{Y_{f}}\right) \cap C^{0, \alpha}\left(\overline{Y_{m}}\right)}\right),
\end{aligned}
$$

where $i \in\{0,1\}, \alpha \in(0,1)$, and $c$ is a constant independent of $\omega$. See (3.1) for $\mathbf{D}_{1}$.

Under A1-A2 and $v \in(0,1]$, the solution of (2.5) satisfies, by Lemma 3.3,

$$
\left\|\mathbb{X}_{v}^{(i)}\right\|_{C^{2}\left(\overline{Y_{f}}\right) \cap C^{2}\left(\overline{Y_{m}}\right)} \leq c,
$$

where $c$ is independent of $v$. Under A1-A2, the solution of (2.6) satisfies, by Theorem 6.30 [16],

$$
\left\|\mathbb{X}_{0}^{(i)}\right\|_{C^{2}\left(\overline{Y_{f}}\right)} \leq c,
$$

where $c$ is a constant. By (3.17) and (3.18), it is not difficult to see that there are positive constants $\omega_{0}, \ell_{5}, \ell_{6}$ such that the symmetric positive definite matrix $\mathcal{K}_{v}$ for $v \in[0,1]$ in (2.9) satisfies

$$
\left\{\begin{array}{l}
\ell_{5} I \leq \mathcal{K}_{v} \leq \ell_{6} I \\
\left|\mathcal{K}_{v}-\mathcal{K}_{0}\right| \leq c v \quad \text { where } c \text { is independent of } v .
\end{array}\right.
$$

Define a part of boundary of $Y$ by $\partial \widetilde{Y}_{n} \equiv\left\{y \in \partial Y \mid y=\left(y_{1}, y_{2}, \cdots, y_{n-1}, 0\right)\right\}$ and consider the following problem

$$
\begin{cases}-\nabla \cdot\left(\mathbb{E}_{\omega^{2}} \mathbf{K} \nabla U_{\omega}+Q_{\omega}\right)+\mathbb{E}_{\omega} \mathbb{P}_{\omega} U_{\omega}=F_{\omega} & \text { in } Y, \\ U_{\omega}=U_{b_{\omega}} & \text { on } \partial \widetilde{Y}_{n} .\end{cases}
$$

Let $Y_{m} \subset \mathbf{D}_{3} \subset Y$ satisfy $\min \left\{\operatorname{dist}\left(Y_{m}, \partial \mathbf{D}_{3}\right)\right.$, dist $\left.\left(\mathbf{D}_{3}, \partial Y \backslash \partial \widetilde{Y}_{n}\right)\right\}>0$. By an analogous argument as that for Lemma 3.2, we see

Lemma 3.4 Under $A 1-A 2, \omega \in(0,1], p \in(n, 6), \mathbf{M}>0$, and $\mathbb{P}_{\omega}(x) \in[0, \mathbf{M}]$ for all $x \in Y$, any solution of (3.20) satisfies

$$
\begin{aligned}
& \left\|\mathbb{E}_{\omega^{3 / 2}} U_{\omega}\right\|_{W^{1, p}\left(\mathbf{D}_{3} \backslash \overline{Y_{m}}\right) \cap W^{1, p}\left(Y_{m}\right)} \leq c\left(\left\|U_{b_{\omega}}\right\|_{W^{1, p}\left(Y_{f}\right)}+\left\|U_{\omega}\right\|_{L^{2}\left(Y_{f}\right)}\right. \\
& \left.+\left\|\mathbb{E}_{1 / \omega} Q_{\omega}\right\|_{L^{2}(Y)}+\left\|\mathbb{E}_{1 / \omega} F_{\omega}\right\|_{H^{-1}(Y)}+\left\|\mathbb{E}_{1 / \sqrt{\omega}} Q_{\omega}\right\|_{L^{p}(Y)}+\left\|\mathbb{E}_{1 / \sqrt{\omega}} F_{\omega}\right\|_{W^{-1, p}(Y)}\right),
\end{aligned}
$$

where $c$ is a constant independent of $\omega$. 
Under A1-A2, $\omega \in(0,1]$, and $p \in(n, \infty)$, any solution of $(3.20)$ with $\mathbb{P}_{\omega}=0$ satisfies

$$
\begin{gathered}
\left\|\mathbb{E}_{\omega} U_{\omega}\right\|_{W^{1, p}\left(\mathbf{D}_{3} \backslash \overline{Y_{m}}\right) \cap W^{1, p}\left(Y_{m}\right)} \leq c\left(\left\|U_{b_{\omega}}\right\|_{W^{1, p}\left(Y_{f}\right)}+\left\|U_{\omega}\right\|_{L^{2}\left(Y_{f}\right)}\right. \\
\left.+\left\|\mathbb{E}_{1 / \omega} Q_{\omega}\right\|_{L^{p}(Y)}+\left\|\mathbb{E}_{1 / \omega} F_{\omega}\right\|_{W^{-1, p}(Y)}\right),
\end{gathered}
$$

where $c$ is a constant independent of $\omega$.

One example below shows that the second-order derivatives of the solution of (1.1) may not be bounded uniformly in $\omega, \epsilon$ in the high-permeability subregion $\Omega_{f}^{\epsilon}$.

Remark 3.1 Assume that $B_{1}(0) \subset \Omega(\omega)$ and $\eta$ is a bell-shaped smooth function satisfying $\eta \in C_{0}^{\infty}\left(B_{1}(0)\right), \eta \in[0,1]$, and $\eta(x)=1$ in $B_{1 / 2}(0)$. Employ $(2.5), \eta$, and $\mathbb{X}_{\omega, \omega}^{(1)}$ for $\omega \in(0,1)$ to obtain

$$
\left\{\begin{array}{cc}
-\nabla \cdot\left(\mathbf{E}_{\omega^{2}, \omega} \mathbf{K}_{\omega}\left(\nabla\left(\eta \mathbb{X}_{\omega, \omega}^{(1)}\right)-\mathbb{X}_{\omega, \omega}^{(1)} \nabla \eta+\eta \vec{e}_{1}\right)\right) & \\
=-\mathbf{E}_{\omega^{2}, \omega} \mathbf{K}_{\omega}\left(\nabla \mathbb{X}_{\omega, \omega}^{(1)}+\vec{e}_{1}\right) \nabla \eta & \text { in } \Omega \\
\eta \mathbb{X}_{\omega, \omega}^{(1)}=0 & \text { on } \partial \Omega
\end{array}\right.
$$

where $\vec{e}_{1}$ is a unit vector in the first coordinate direction. By (3.17), we see that

$$
\left\|\mathbb{X}_{\omega, \omega}^{(1)} \nabla \eta-\eta \vec{e}_{1}\right\|_{W^{1, \infty}\left(B_{1}(0)\right)}+\left\|\left(\nabla \mathbb{X}_{\omega, \omega}^{(1)}+\vec{e}_{1}\right) \nabla \eta\right\|_{L^{\infty}\left(B_{1}(0)\right)}
$$

is bounded uniformly in $\omega$, but $\left\|\eta \mathbb{X}_{\omega, \omega}^{(1)}\right\|_{W^{2, p}\left(B_{1}(0) \cap \Omega_{f}^{\omega}\right)}$ for $p \in[1, \infty]$ is not bounded uniformly in $\omega$.

\section{Uniform Hölder estimate}

A1-A2 are assumed in this section. We shall derive uniform Hölder estimates for non-uniform elliptic equations, that is, Theorem 2.1 and Theorem 2.2. The Hölder estimate in the interior region is considered in Sect. 4.1, and the estimate around the boundary is in Sect. 4.2.

\subsection{Interior estimate}

For convenience, we let $\overline{B_{1}(0)} \subset \Omega$.

Lemma 4.1 For any $\delta, \mathbf{M}>0$, there are $\theta_{1}, \theta_{2} \in(0,1)$ (depending on $\delta, \mathbf{M}, \mathbf{K}, Y_{f}$ ) with $\theta_{1}<\theta_{2}^{2}$ and there is a $\omega_{0} \in(0,1)$ (depending on $\left.\theta_{1}, \theta_{2}, \delta, \mathbf{M}, \mathbf{K}\right)$ such that if

$$
-\nabla \cdot\left(\mathbf{E}_{\omega^{2}, \nu} \mathbf{K}_{v} \nabla \mathbb{U}_{\omega, v}+\mathbb{Q}_{\omega, v}\right)+\mathbf{E}_{\omega, v} \mathbb{P}_{\omega, \nu} \mathbb{U}_{\omega, v}=\mathbb{F}_{\omega, v} \quad \text { in } B_{1}(0),
$$

and if

$$
\left\{\begin{array}{c}
\omega, v \in\left(0, \omega_{0}\right), \quad \theta \in\left[\theta_{1}, \theta_{2}\right], \quad \mathbb{P}_{\omega, v}(x) \in[0, \mathbf{M}] \text { for all } x \in B_{1}(0), \\
\max \left\{\left\|\mathbf{E}_{\omega, v} \mathbb{U}_{\omega, v}\right\|_{L^{2}\left(B_{1}(0)\right)},\left\|\omega_{0}^{-1} \mathbb{Q}_{\omega, \nu} \mathcal{X}_{\Omega_{f}^{v}}+\omega^{-1} \mathbb{Q}_{\omega, \nu} \mathcal{X}_{\Omega_{m}^{v}}\right\|_{L^{n+\delta}\left(B_{1}(0)\right)},\right. \\
\left.\omega_{0}^{-1}\left\|\mathbb{F}_{\omega, \nu} \mathcal{X}_{\Omega_{f}^{v}}+\omega^{-1} \max \{\omega, v\} \mathbb{F}_{\omega, \nu} \mathcal{X}_{\Omega_{m}^{v}}\right\|_{L^{n+\delta}\left(B_{1}(0)\right)}\right\} \leq 1,
\end{array}\right.
$$

then

$$
\left\{\begin{array}{l}
f_{B_{\theta}(0)}\left|\Pi_{v} \mathbb{U}_{\omega, v}\right|_{\Omega_{f}^{v}}-\left.\left(\left.\Pi_{v} \mathbb{U}_{\omega, v}\right|_{\Omega_{f}^{v}}\right)_{0, \theta}\right|^{2} d x \leq \theta^{2 \mu} \\
f_{B_{\theta}(0) \cap \Omega_{m}^{v}} \omega^{2}\left|\mathbb{U}_{\omega, v}-\left(\left.\Pi_{v} \mathbb{U}_{\omega, v}\right|_{\Omega_{f}^{v}}\right)_{0, \theta}\right|^{2} d x \leq \theta^{2 \mu}
\end{array}\right.
$$


where $\mu \equiv \frac{\delta}{n+\delta}$. See Sect. 2 for $\left(\left.\Pi_{\nu} \mathbb{U}_{\omega, \nu}\right|_{\Omega_{f}^{v}}\right)_{0, \theta}$, the average value of the extension function $\left.\Pi_{\nu} \mathbb{U}_{\omega, v}\right|_{\Omega_{f}^{v}}$ in $B_{\theta}(0)$.

Proof Consider the following problem

$$
-\nabla \cdot\left(\mathcal{K}_{0} \nabla \mathbb{U}\right)+\mathbb{P U}=0 \quad \text { in } B_{3 / 4}(0),
$$

where $\mathcal{K}_{0}$ is defined in (2.9) and $\mathbb{P}(x) \in[0, \mathbf{M}]$ for $x \in B_{3 / 4}(0)$. Any solution $\mathbb{U}$ of (4.4) satisfies, by Theorem 9.11 [16] and (3.19),

$$
\|\mathbb{U}\|_{C^{1, \alpha}\left(\overline{B_{1 / 2}(0)}\right)} \leq c\|\mathbb{U}\|_{L^{2}\left(B_{3 / 4}(0)\right)},
$$

where $\alpha \in(0,1)$ and $c$ only depends on $\mathcal{K}_{0}$, M. If $\check{\mu}$ satisfies $\mu<\check{\mu}<1$, then, by Theorem 1.2 in page 70 [15],

$$
f_{B_{\theta}(0)}\left|\mathbb{U}-(\mathbb{U})_{0, \theta}\right|^{2} \mathrm{~d} x \leq \theta^{2 \check{\mu}} f_{B_{3 / 4}(0)} \mathbb{U}^{2} \mathrm{~d} x
$$

for $\theta$ (depending on $\delta, \mathcal{K}_{0}, \mathbf{M}$ ) sufficiently small. Let us fix $\theta_{1}, \theta_{2} \in\left(0, \frac{1}{2}\right)$ so that $\theta_{1}<\theta_{2}^{2}$ and (4.5) holds for any $\theta \in\left[\theta_{1}, \theta_{2}\right]$.

Now we claim (4.3) $)_{1}$. If not, there is a sequence $\left\{\theta_{\omega, \nu}, \mathbb{P}_{\omega, \nu}, \mathbb{U}_{\omega, \nu}, \mathbb{Q}_{\omega, \nu}, \mathbb{F}_{\omega, \nu}\right\}$ satisfying (4.1) and, as $\omega, v \rightarrow 0$,

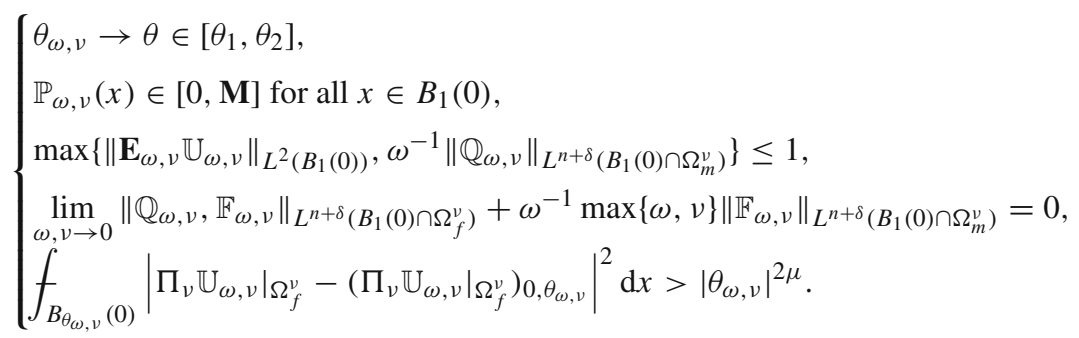

By energy method and A2, there is a constant $c$ independent of $\omega, v$ such that

$$
\left\|\mathbb{U}_{\omega, v}\right\|_{H^{1}\left(B_{4 / 5}(0) \cap \Omega_{f}^{v}\right)}+\left\|\omega \nabla \mathbb{U}_{\omega, \nu}, \omega^{1 / 2} \mathbb{P}_{\omega, \nu}^{1 / 2} \mathbb{U}_{\omega, v}\right\|_{L^{2}\left(B_{4 / 5}(0) \cap \Omega_{m}^{v}\right)} \leq c .
$$

By compactness principle and by tracing the proof of Theorem 2.3 [3], we can extract a subsequence (same notation for subsequence) such that

$$
\left\{\begin{array}{lll}
\left.\Pi_{\nu} \mathbb{U}_{\omega, \nu}\right|_{\Omega_{f}^{v}} \rightarrow \mathbb{U} & \text { in } L^{2}\left(B_{3 / 4}(0)\right) \text { strongly } & \\
\mathbf{E}_{\omega^{2}, \nu} \mathbf{K}_{v} \nabla \mathbb{U}_{\omega, \nu} \rightarrow \mathcal{K}_{0} \nabla \mathbb{U} & \text { in } L^{2}\left(B_{3 / 4}(0)\right) \text { weakly } \\
\mathbf{E}_{\omega, \nu} \mathbb{P}_{\omega, \nu} \mathbb{U}_{\omega, \nu} \rightarrow \mathbb{P} & \text { in } L^{2}\left(B_{3 / 4}(0)\right) \text { weakly }
\end{array}\right.
$$

where $\mathbb{P}(x) \in[0, \mathbf{M}]$ for all $x \in B_{3 / 4}(0), \mathcal{K}_{0}$ is a constant symmetric positive definite matrix, $\ell_{5} \leq \mathcal{K}_{0} \leq \ell_{6}$, and $\ell_{5}, \ell_{6}$ are positive constants (see (2.9) and (3.19)). The $\mathbb{U}$ in (4.7) satisfies (4.4). Equations (4.5)-(4.7) then imply

$$
\begin{aligned}
\theta^{2 \mu} & =\lim _{\omega, \nu \rightarrow 0}\left|\theta_{\omega, v}\right|^{2 \mu} \leq \lim _{\omega, v \rightarrow 0} f_{B_{\theta_{\omega, v}}(0)}\left|\Pi_{\nu} \mathbb{U}_{\omega, \nu}\right|_{\Omega_{f}^{v}}-\left.\left(\left.\Pi_{\nu} \mathbb{U}_{\omega, v}\right|_{\Omega_{f}^{v}}\right)_{0, \theta_{\omega, v}}\right|^{2} \\
& =f_{B_{\theta}(0)} \mathbb{U}^{2}-\left|f_{B_{\theta}(0)} \mathbb{U}\right|^{2}=f_{B_{\theta}(0)}\left|\mathbb{U}-(\mathbb{U})_{0, \theta}\right|^{2} \leq \theta^{2 \check{\mu}} f_{B_{3 / 4}(0)} \mathbb{U}^{2} \mathrm{~d} x .
\end{aligned}
$$

If $\theta_{2}$ is small enough, then we get contradiction. Therefore, we prove (4.3) 1 . 
Set $\zeta \equiv \theta^{-\mu}\left(\left.\Pi_{\nu} \mathbb{U}_{\omega, v}\right|_{\Omega_{f}^{v}}-\left(\left.\Pi_{\nu} \mathbb{U}_{\omega, v}\right|_{\Omega_{f}^{v}}\right)_{0, \theta}\right)$ and $\eta \equiv \theta^{-\mu}\left(\mathbb{U}_{\omega, v}-\left(\left.\Pi_{\nu} \mathbb{U}_{\omega, v}\right|_{\Omega_{f}^{v}}\right)_{0, \theta}\right)$. (4.1) implies, for any smooth function $\varphi$ with support in $v\left(Y_{m}+j\right) \subset B_{\theta}(0) \cap \Omega_{m}^{v}$ for some $j \in \mathbb{Z}^{n}$,

$$
\begin{aligned}
& \omega^{2} \int_{\nu\left(Y_{m}+j\right)}(\eta-\zeta) \nabla \cdot\left(\mathbf{K}_{v} \nabla \varphi\right)-\omega \int_{\nu\left(Y_{m}+j\right)} \mathbb{P}_{\omega, v}(\eta-\zeta) \varphi \\
& \quad=\int_{\nu\left(Y_{m}+j\right)}\left(\omega^{2} \mathbf{K}_{v} \nabla \zeta+\theta^{-\mu} \mathbb{Q}_{\omega, \nu}\right) \nabla \varphi+\theta^{-\mu}\left(\left.\omega \mathbb{P}_{\omega, v} \Pi_{\nu} \mathbb{U}_{\omega, v}\right|_{\Omega_{f}^{\nu}}-\mathbb{F}_{\omega, v}\right) \varphi .
\end{aligned}
$$

If $\varphi$ is the solution of

$$
\begin{cases}-\omega^{2} \nabla \cdot\left(\mathbf{K}_{v} \nabla \varphi\right)+\omega \mathbb{P}_{\omega, \nu} \varphi=\zeta-\eta & \text { in } v\left(Y_{m}+j\right), \\ \varphi=0 & \text { on } v\left(\partial Y_{m}+j\right),\end{cases}
$$

then $c_{1} v^{-1} \omega^{2}\|\varphi\|_{L^{2}\left(v\left(Y_{m}+j\right)\right)} \leq \omega^{2}\|\nabla \varphi\|_{L^{2}\left(v\left(Y_{m}+j\right)\right)} \leq c_{2} v\|\eta-\zeta\|_{L^{2}\left(v\left(Y_{m}+j\right)\right)}$, where $c_{1}, c_{2}$ are independent of $v$. (4.8) and (4.9) imply

$$
\begin{aligned}
& \int_{\nu\left(Y_{m}+j\right)} \omega^{2}|\eta-\zeta|^{2} \leq c \int_{\nu\left(Y_{m}+j\right)} \omega^{2} v^{2}|\nabla \zeta|^{2} \\
& \quad+c \int_{\nu\left(Y_{m}+j\right)} v^{2} \theta^{-2 \mu}\left(\left|\omega^{-1} \mathbb{Q} \omega, v\right|^{2}+\left.v^{2} \mathbb{P}_{\omega, \nu}^{2}\left|\Pi_{v} \mathbb{U}_{\omega, v}\right| \Omega_{f}^{\nu}\right|^{2}+\left|v \omega^{-1} \mathbb{F}_{\omega, \nu}\right|^{2}\right) .
\end{aligned}
$$

Summing (4.10) over all $v\left(Y_{m}+j\right) \subset B_{\theta}(0) \cap \Omega_{m}^{v}$ for $j \in \mathbb{Z}^{n}$, we obtain (4.3) 2 if $\omega_{0}$ is small enough.

Lemma 4.2 For any $\delta \in(0,3)$ and $\mathbf{M}>0$, there are $\theta_{1}, \theta_{2} \in(0,1)$ (depending on $\delta, \mathbf{M}, \mathbf{K}, Y_{f}$ ) with $\theta_{1}<\theta_{2}^{2}$ and there is a $\omega_{0}>0$ (depending on $\theta_{1}, \theta_{2}, \delta, \mathbf{M}, \mathbf{K}$ ) such that if

$$
-\nabla \cdot\left(\mathbf{E}_{\omega^{2}, \epsilon} \mathbf{K}_{\epsilon} \nabla U_{\omega, \epsilon}+Q_{\omega, \epsilon}\right)+\gamma \mathbf{E}_{\omega, \epsilon} \mathbf{P}_{\omega, \epsilon} U_{\omega, \epsilon}=F_{\omega, \epsilon} \quad \text { in } B_{1}(0),
$$

and if $\omega, \epsilon \in\left(0, \omega_{0}\right), \theta \in\left[\theta_{1}, \theta_{2}\right], \gamma \in[0,1], \mathbf{P}_{\omega, \epsilon}(x) \in[0, \mathbf{M}]$ for all $x \in B_{1}(0)$, and $k$ satisfying $\epsilon / \theta^{k} \leq \omega_{0}$, then

$$
\left\{\begin{array}{l}
f_{B_{\theta^{k}}(0)}\left|\Pi_{\epsilon} U_{\omega, \epsilon}\right| \Omega_{f}^{\epsilon}-\left.\left(\Pi_{\epsilon} U_{\omega, \epsilon} \mid \Omega_{f}^{\epsilon}\right)_{0, \theta^{k}}\right|^{2} d x \leq \theta^{2 k \mu}\left|J_{\omega, \epsilon}\right|^{2}, \\
f_{B_{\theta^{k}}(0) \cap \Omega_{m}^{\epsilon}} \omega^{2}\left|U_{\omega, \epsilon}-\left(\Pi_{\epsilon} U_{\omega, \epsilon} \mid \Omega_{f}^{\epsilon}\right)_{0, \theta^{k}}\right|^{2} d x \leq \theta^{2 k \mu}\left|J_{\omega, \epsilon}\right|^{2},
\end{array}\right.
$$

where $\mu \equiv \frac{\delta}{n+\delta}$ and

$$
\begin{aligned}
J_{\omega, \epsilon} \equiv & 3 \omega_{0}^{-1}\left(\left\|\mathbf{E}_{\omega, \epsilon} U_{\omega, \epsilon}\right\|_{L^{2}\left(B_{1}(0)\right)}+\omega^{-1}\left\|Q_{\omega, \epsilon}, \max \{\omega, \epsilon\} F_{\omega, \epsilon}\right\|_{L^{n+\delta}\left(B_{1}(0) \cap \Omega_{m}^{\epsilon}\right)}\right. \\
& \left.+\left\|Q_{\omega, \epsilon}, F_{\omega, \epsilon}\right\|_{L^{n+\delta\left(B_{1}(0) \cap \Omega_{f}^{\epsilon}\right)}}\right) .
\end{aligned}
$$

Proof Let $c$ denote a constant independent of $\omega, \epsilon, \gamma$. This proof is done by induction. For $k=1$, we define $\mathbb{U}_{\omega, \epsilon} \equiv \frac{U_{\omega, \epsilon}}{J_{\omega, \epsilon}}, \mathbb{Q}_{\omega, \epsilon} \equiv \frac{Q_{\omega, \epsilon}}{J_{\omega, \epsilon}}, \mathbb{F}_{\omega, \epsilon} \equiv \frac{F_{\omega, \epsilon}}{J_{\omega, \epsilon}}, \mathbb{P}_{\omega, \epsilon} \equiv \gamma \mathbf{P}_{\omega, \epsilon}$. Then they satisfy (4.1) and (4.2) with $v=\epsilon$. By Lemma 4.1,

$$
\left\{\begin{array}{l}
f_{B_{\theta}(0)}\left|\Pi_{\epsilon} \mathbb{U}_{\omega, \epsilon}\right| \Omega_{f}^{\epsilon}-\left.\left(\left.\Pi_{\epsilon} \mathbb{U}_{\omega, \epsilon}\right|_{\Omega_{f}^{\epsilon}}\right)_{0, \theta}\right|^{2} \mathrm{~d} x \leq \theta^{2 \mu}, \\
f_{B_{\theta}(0) \cap \Omega_{m}^{\epsilon}} \omega^{2}\left|\mathbb{U}_{\omega, \epsilon}-\left(\left.\Pi_{\epsilon} \mathbb{U}_{\omega, \epsilon}\right|_{\Omega_{f}^{\epsilon}}\right)_{0, \theta}\right|^{2} \mathrm{~d} x \leq \theta^{2 \mu} .
\end{array}\right.
$$


This implies (4.12) for $k=1$. By energy method and A2, any solution of (4.11) satisfies

$$
\begin{gathered}
\left\|U_{\omega, \epsilon}\right\|_{H^{1}\left(B_{4 / 5}(0) \cap \Omega_{f}^{\epsilon}\right)} \leq c\left(\left\|\mathbf{E}_{\omega, \epsilon} U_{\omega, \epsilon}\right\|_{L^{2}\left(B_{1}(0)\right)}+\left\|\mathbf{E}_{1 / \omega, \epsilon} Q_{\omega, \epsilon}\right\|_{L^{2}\left(B_{1}(0)\right)}\right. \\
\left.+\left\|F_{\omega, \epsilon}\right\|_{L^{2}\left(B_{1}(0)\right)}+\epsilon \omega^{-1}\left\|F_{\omega, \epsilon}\right\|_{L^{2}\left(B_{1}(0) \cap \Omega_{m}^{\epsilon}\right)}\right) \equiv c \hat{\mathcal{I}}
\end{gathered}
$$

By Theorem 7.26 [16] and Remark 2.1,

$$
\left\|\left.\Pi_{\epsilon} U_{\omega, \epsilon}\right|_{\Omega_{f}^{\epsilon}}\right\|_{L^{\frac{2 n}{n-2}\left(B_{4 / 5}(0)\right)}} \leq c \hat{\mathcal{I}}
$$

Suppose (4.12) holds for some $k$ satisfying $\epsilon / \theta^{k} \leq \omega_{0}$, we define, in $B_{1}(0) \backslash \partial \Omega_{m}^{\epsilon} / \theta^{k}$,

$$
\left\{\begin{array}{l}
\mathbb{U}_{\omega, \epsilon / \theta^{k}}(x) \equiv J_{\omega, \epsilon}^{-1} \theta^{-k \mu}\left(U_{\omega, \epsilon}\left(\theta^{k} x\right)-\left(\left.\Pi_{\epsilon} U_{\omega, \epsilon}\right|_{\Omega_{f}^{\epsilon}}\right)_{0, \theta^{k}}\right), \\
\mathbb{Q}_{\omega, \epsilon / \theta^{k}}(x) \equiv J_{\omega, \epsilon}^{-1} \theta^{k(1-\mu)} Q_{\omega, \epsilon}\left(\theta^{k} x\right), \\
\mathbb{F}_{\omega, \epsilon / \theta^{k}}(x) \equiv J_{\omega, \epsilon}^{-1} \theta^{k(2-\mu)}\left(F_{\omega, \epsilon}\left(\theta^{k} x\right)-\gamma \mathbf{E}_{\omega, \epsilon / \theta^{k}}(x) \mathbf{P}_{\omega, \epsilon}\left(\theta^{k} x\right)\left(\left.\Pi_{\epsilon} U_{\omega, \epsilon}\right|_{\Omega_{f}^{\epsilon}}\right)_{0, \theta^{k}}\right), \\
\mathbb{P}_{\omega, \epsilon / \theta^{k}}(x) \equiv \theta^{2 k} \gamma \mathbf{P}_{\omega, \epsilon}\left(\theta^{k} x\right) .
\end{array}\right.
$$

Then they satisfy

$$
-\nabla \cdot\left(\mathbf{E}_{\omega^{2}, \epsilon / \theta^{k}} \mathbf{K}_{\epsilon / \theta^{k}} \nabla \mathbb{U}_{\omega, \epsilon / \theta^{k}}+\mathbb{Q}_{\omega, \epsilon / \theta^{k}}\right)+\mathbf{E}_{\omega, \epsilon / \theta^{k}} \mathbb{P}_{\omega, \epsilon / \theta^{k}} \mathbb{U}_{\omega, \epsilon / \theta^{k}}=\mathbb{F}_{\omega, \epsilon / \theta^{k}} \quad \text { in } B_{1}(0) .
$$

By triangle inequality,

$$
\left\{\begin{array}{l}
\left\|\mathbb{F}_{\omega, \epsilon / \theta^{k}}\right\|_{L^{n+\delta}\left(B_{1}(0) \cap \Omega_{f}^{\epsilon} / \theta^{k}\right)} \\
\quad \leq \frac{\theta^{k}}{J_{\omega, \epsilon}}\left\|F_{\omega, \epsilon}\right\|_{L^{n+\delta}\left(B_{\theta^{k}}(0) \cap \Omega_{f}^{\epsilon}\right)}+\frac{\theta^{k(3-\mu-n / 2)} \mathbf{M}}{J_{\omega, \epsilon}}\left\|\left.\Pi_{\epsilon} U_{\omega, \epsilon}\right|_{\Omega_{f}^{\epsilon}}\right\|_{L^{2 n /(n-2)}\left(B_{\theta^{k}}(0)\right)}, \\
\left\|\mathbb{F}_{\omega, \epsilon / \theta^{k}}\right\|_{L^{n+\delta}\left(B_{1}(0) \cap \Omega_{m}^{\epsilon} / \theta^{k}\right)} \\
\quad \leq \frac{\theta^{k}}{J_{\omega, \epsilon}}\left\|F_{\omega, \epsilon}\right\|_{L^{n+\delta}\left(B_{\theta^{k}}(0) \cap \Omega_{m}^{\epsilon}\right)}+\frac{\omega \theta^{k(3-\mu-n / 2)} \mathbf{M}}{J_{\omega, \epsilon}}\left\|\left.\Pi_{\epsilon} U_{\omega, \epsilon}\right|_{\Omega_{f}^{\epsilon}}\right\|_{L^{2 n /(n-2)\left(B_{\theta^{k}}(0)\right)}} .
\end{array}\right.
$$

By induction, (4.13), and small $\theta$,

$$
\left\{\begin{array}{l}
\mathbb{P}_{\omega, \epsilon / \theta^{k}}(x) \in[0, \mathbf{M}] \text { for all } x \in B_{1}(0) \\
\left\|\mathbf{E}_{\omega, \epsilon / \theta^{k}} \mathbb{U}_{\omega, \epsilon / \theta^{k}}\right\|_{L^{2}\left(B_{1}(0)\right)} \leq 1 \\
\left\|\omega_{0}^{-1} \mathbb{Q}_{\omega, \epsilon / \theta^{k}} \mathcal{X}_{\Omega_{f}^{\epsilon} / \theta^{k}}+\omega^{-1} \mathbb{Q}_{\omega, \epsilon / \theta^{k}} \mathcal{X}_{\Omega_{m}^{\epsilon} / \theta^{k}}\right\|_{L^{n+\delta}\left(B_{1}(0)\right)} \leq 1 \\
\omega_{0}^{-1}\left\|\mathbb{F}_{\omega, \epsilon / \theta^{k}} \mathcal{X}_{\Omega_{f}^{\epsilon} / \theta^{k}}+\omega^{-1} \max \left\{\omega, \epsilon / \theta^{k}\right\} \mathbb{F}_{\omega, \epsilon / \theta^{k}} \mathcal{X}_{\Omega_{m}^{\epsilon} / \theta^{k}}\right\|_{L^{n+\delta}\left(B_{1}(0)\right)} \leq 1
\end{array}\right.
$$

By Lemma 4.1 (take $\left.v=\epsilon / \theta^{k}\right)$, we obtain

$$
\left\{\begin{array}{l}
f_{B_{\theta}(0)}\left|\Pi_{\epsilon / \theta^{k}} \mathbb{U}_{\omega, \epsilon / \theta^{k}}\right|_{\Omega_{f}^{\epsilon} / \theta^{k}}-\left.\left(\left.\Pi_{\epsilon / \theta^{k}} \mathbb{U}_{\omega, \epsilon / \theta^{k}}\right|_{\Omega_{f}^{\epsilon} / \theta^{k}}\right)_{0, \theta}\right|^{2} \mathrm{~d} x \leq \theta^{2 \mu}, \\
f_{B_{\theta}(0) \cap \Omega_{m}^{\epsilon} / \theta^{k}} \omega^{2}\left|\mathbb{U}_{\omega, \epsilon / \theta^{k}}-\left(\left.\Pi_{\epsilon / \theta^{k}} \mathbb{U}_{\omega, \epsilon / \theta^{k}}\right|_{\Omega_{f}^{\epsilon} / \theta^{k}}\right)_{0, \theta}\right|^{2} \mathrm{~d} x \leq \theta^{2 \mu} .
\end{array}\right.
$$


Note, by Remark 2.1,

$$
\left\{\begin{array}{c}
f_{B_{\theta}(0)}\left|\Pi_{\epsilon / \theta^{k}} \mathbb{U}_{\omega, \epsilon / \theta^{k}}\right|_{\Omega_{f}^{\epsilon} / \theta^{k}}-\left.\left(\left.\Pi_{\epsilon / \theta^{k}} \mathbb{U}_{\omega, \epsilon / \theta^{k}}\right|_{\Omega_{f}^{\epsilon} / \theta^{k}}\right)_{0, \theta}\right|^{2} \mathrm{~d} x \\
=f_{B_{\theta^{k+1}}(0)} \frac{\left|\Pi_{\epsilon} U_{\omega, \epsilon}\right|_{\Omega_{f}^{\epsilon}}-\left.\left(\left.\Pi_{\epsilon} U_{\omega, \epsilon}\right|_{\Omega_{f}^{\epsilon}}\right)_{0, \theta^{k+1}}\right|^{2}}{\left|J_{\omega, \epsilon}\right|^{2} \theta^{2 k \mu}} \mathrm{d} x, \\
f_{B_{\theta}(0) \cap \Omega_{m}^{\epsilon} / \theta^{k}}\left|\mathbb{U}_{\omega, \epsilon / \theta^{k}}-\left(\left.\Pi_{\epsilon / \theta^{k}} \mathbb{U}_{\omega, \epsilon / \theta^{k}}\right|_{\Omega_{f}^{\epsilon} / \theta^{k}}\right)_{0, \theta}\right|^{2} \mathrm{~d} x \\
=f_{B_{\theta^{k+1}}(0) \cap \Omega_{m}^{\epsilon}} \frac{\left|U_{\omega, \epsilon}-\left(\Pi_{\epsilon} U_{\omega, \epsilon} \mid \Omega_{f}^{\epsilon}\right)_{0, \theta^{k+1}}\right|^{2}}{\left|J_{\omega, \epsilon}\right|^{2} \theta^{2 k \mu}} \mathrm{d} x .
\end{array}\right.
$$

Equations (4.14)-(4.15) imply the inequality (4.12) for $k+1$ case.

Lemma 4.3 For any $\delta \in(0,3)$ and $\mathbf{M}>0$, there is a $\omega_{*} \in(0,1)$ (depending on $\left.\delta, \mathbf{M}, \mathbf{K}, Y_{f}\right)$ such that if $\omega, \epsilon \in\left(0, \omega_{*}\right), \gamma \in[0,1]$, and $\mathbf{P}_{\omega, \epsilon}(x) \in[0, \mathbf{M}]$ for all $x \in B_{1}(0)$, then any solution of (4.11) satisfies

$$
\left[U_{\omega, \epsilon}\right]_{C^{0, \mu}\left(\overline{B_{1 / 2}(0) \cap \Omega_{f}^{\epsilon}}\right)}+\sup _{\substack{j \in \mathbb{Z}^{n} \\ \epsilon\left(Y_{m}+j\right) \subset B_{1 / 2}(0) \cap \Omega_{m}^{\epsilon}}} \omega^{3 / 2}\left[U_{\omega, \epsilon}\right]_{C^{0, \mu}\left(\epsilon\left(\overline{Y_{m}}+j\right)\right)} \leq c J_{\omega, \epsilon},
$$

where $c$ is a constant independent of $\omega, \epsilon, \gamma$. See Lemma 4.2 for $\mu, J_{\omega, \epsilon}$.

Proof Let $\theta_{1}, \theta_{2}, \omega_{0}$ be same as those in Lemma 4.2, define $\omega_{*} \equiv \omega_{0} \theta_{2} / 2$, and let $\omega, \epsilon \leq \omega_{*}$. Denote by $c$ a constant independent of $\omega, \epsilon, \gamma$. Because of $\theta_{1}<\theta_{2}^{2}$, for any $r \in\left[\epsilon / \omega_{0}, \theta_{2}\right]$, there are $\theta \in\left[\theta_{1}, \theta_{2}\right]$ and $k \in \mathbb{N}$ satisfying $r=\theta^{k}$. Lemma 4.2 implies, for any $r \in\left[\epsilon / \omega_{0}, \theta_{2}\right]$,

$$
\left\{\begin{array}{l}
f_{B_{r}(0)}\left|\Pi_{\epsilon} U_{\omega, \epsilon}\right| \Omega_{f}^{\epsilon}-\left.\left(\left.\Pi_{\epsilon} U_{\omega, \epsilon}\right|_{\Omega_{f}^{\epsilon}}\right)_{0, r}\right|^{2} \mathrm{~d} x \leq r^{2 \mu}\left|J_{\omega, \epsilon}\right|^{2}, \\
f_{B_{r}(0) \cap \Omega_{m}^{\epsilon}} \omega^{2}\left|U_{\omega, \epsilon}-\left(\left.\Pi_{\epsilon} U_{\omega, \epsilon}\right|_{\Omega_{f}^{\epsilon}}\right)_{0, r}\right|^{2} \mathrm{~d} x \leq r^{2 \mu}\left|J_{\omega, \epsilon}\right|^{2} .
\end{array}\right.
$$

Now we define, in $B_{2 / \omega_{0}}(0) \backslash \partial \Omega_{m}^{\epsilon} / \epsilon$,

$$
\left\{\begin{array}{l}
\mathbb{U}_{\omega, 1}(x) \equiv J_{\omega, \epsilon}^{-1} \epsilon^{-\mu}\left(U_{\omega, \epsilon}(\epsilon x)-\left(\left.\Pi_{\epsilon} U_{\omega, \epsilon}\right|_{\Omega_{f}^{\epsilon}}\right)_{0,2 \epsilon / \omega_{0}}\right) \\
\mathbb{Q}_{\omega, 1}(x) \equiv J_{\omega, \epsilon}^{-1} \epsilon^{1-\mu} Q_{\omega, \epsilon}(\epsilon x) \\
\mathbb{F}_{\omega, 1}(x) \equiv J_{\omega, \epsilon}^{-1} \epsilon^{2-\mu}\left(F_{\omega, \epsilon}(\epsilon x)-\gamma \mathbf{E}_{\omega, 1}(x) \mathbf{P}_{\omega, \epsilon}(\epsilon x)\left(\left.\Pi_{\epsilon} U_{\omega, \epsilon}\right|_{\Omega_{f}^{\epsilon}}\right)_{0,2 \epsilon / \omega_{0}}\right) \\
\mathbb{P}_{\omega, 1} \equiv \epsilon^{2} \gamma \mathbf{P}_{\omega, \epsilon}(\epsilon x)
\end{array}\right.
$$

Then they satisfy

$$
-\nabla \cdot\left(\mathbf{E}_{\omega^{2}, 1} \mathbf{K} \nabla \mathbb{U}_{\omega, 1}+\mathbb{Q}_{\omega, 1}\right)+\mathbf{E}_{\omega, 1} \mathbb{P}_{\omega, 1} \mathbb{U}_{\omega, 1}=\mathbb{F}_{\omega, 1} \quad \text { in } B_{\frac{2}{\omega_{0}}}(0) .
$$

Take $r=\frac{2 \epsilon}{\omega_{0}}$ in (4.17) to get

$$
\left\{\begin{array}{l}
\mathbb{P}_{\omega, 1} \in[0, \mathbf{M}] \text { for all } x \in B_{2 / \omega_{0}}(0), \\
\left\|\mathbf{E}_{\omega, 1} \mathbb{U}_{\omega, 1}\right\|_{L^{2}\left(B_{2 / \omega_{0}}(0)\right)}+\left\|\mathbf{E}_{1 / \omega, 1} \mathbb{Q}_{\omega, 1}, \mathbf{E}_{1 / \omega, 1} \mathbb{F}_{\omega, 1}\right\|_{L^{n+\delta}\left(B_{2 / \omega_{0}}(0)\right)} \leq c .
\end{array}\right.
$$

By (3.3) of Lemma 3.2,

$$
\left[\mathbb{U}_{\omega, 1}\right]_{C^{0, \mu}\left(\overline{B_{1 / \omega_{0}}(0) \cap \Omega_{f}^{\epsilon} / \epsilon}\right)}+\omega^{3 / 2}\left[\mathbb{U}_{\omega, 1}\right]_{C^{0, \mu}\left(\overline{B_{1 / \omega_{0}}(0) \cap \Omega_{m}^{\epsilon} / \epsilon}\right)} \leq c .
$$


(4.18) implies that (4.17) 1 also holds for $r \leq \epsilon / \omega_{0}$. So (4.17) 1 holds for $r \leq \theta_{2}$. Next we shift the origin of the coordinate system to any point $z \in B_{1 / 2}(0)$ and repeat above argument to see that (4.17) $)_{1}$ with 0 replaced by any $z \in B_{1 / 2}(0)$ also holds for $r \in\left(0, \theta_{2}\right)$. Together with Theorem 1.2 in page 70 [15], we obtain the Hölder estimate of $\Pi_{\epsilon} U_{\omega, \epsilon}$ in $B_{1 / 2}(0)$. Hölder estimate of $U_{\omega, \epsilon}$ in $\epsilon\left(\overline{Y_{m}}+j\right) \subset B_{1 / 2}(0) \cap \overline{\Omega_{m}^{\epsilon}}$ is from (4.18). So (4.16) is proved.

Remark 4.1 Let $\omega_{*}$ be same as that in Lemma 4.3. By (3.3) of Lemma 3.2 with $p=n+\delta$, we know that if $\delta \in(0,3), \mathbf{M}>0, \epsilon \in\left[\omega_{*}, 1\right], \omega \in\left(0, \omega_{*}\right), \gamma \in[0,1]$, and $\mathbf{P}_{\omega, \epsilon}(x) \in[0, \mathbf{M}]$ for all $x \in B_{1}(0)$, any solution of (4.11) satisfies (4.16). Together with Lemma 4.3, we know that any solution of (4.11) satisfies (4.16) if $\delta \in(0,3), \mathbf{M}>0, \epsilon \in(0,1), \omega \in\left(0, \omega_{*}\right)$, $\gamma \in[0,1]$, and $\mathbf{P}_{\omega, \epsilon}(x) \in[0, \mathbf{M}]$ for all $x \in B_{1}(0)$.

Let us consider the solutions of (4.11) with $\mathbf{P}_{\omega, \epsilon}=0$. By tracing the arguments of Lemma 4.2, Lemma 4.3, and Remark 4.1 as well as employing (3.16) of Lemma 3.3, then we have

Lemma 4.4 For any $\delta>0$, there is a $\omega_{*}>0$ (depending on $\left.\delta, \mathbf{K}, Y_{f}\right)$ such that if $\omega \in\left(0, \omega_{*}\right)$ and $\epsilon \in(0,1)$, then any solution of (4.11) with $\mathbf{P}_{\omega, \epsilon}=0$ satisfies

$$
\left[U_{\omega, \epsilon}\right]_{C^{0, \mu}\left(\overline{B_{1 / 2}(0) \cap \Omega_{f}^{\epsilon}}\right)}+\sup _{\substack{j \in \mathbb{Z}^{n} \\ \epsilon\left(Y_{m}+j\right) \subset B_{1 / 2}(0) \cap \Omega_{m}^{\epsilon}}} \omega\left[U_{\omega, \epsilon}\right]_{C^{0, \mu}\left(\epsilon\left(\overline{Y_{m}}+j\right)\right)} \leq c J_{\omega, \epsilon},
$$

where $c$ is a constant independent of $\omega, \epsilon$. See Lemma 4.2 for $\mu, J_{\omega, \epsilon}$.

\subsection{Boundary estimate}

In this subsection, we assume $0 \in \partial \Omega$. By A1, there is a $C^{2,1}$ function $\rho: \mathbb{R}^{n-1} \rightarrow \mathbb{R}$ satisfying

$$
\left\{\begin{array}{l}
\rho(0)=|\nabla \rho(0)|=0, \\
B_{1}(0) \cap \Omega / t=B_{1}(0) \cap\left\{\left(x^{\prime}, x_{n}\right) \in \mathbb{R}^{n} \mid t x_{n}>\rho\left(t x^{\prime}\right)\right\} \quad \text { if } t \in(0,1] .
\end{array}\right.
$$

If $t=0$, we define $B_{1}(0) \cap \Omega / t \equiv B_{1}(0) \cap\left\{\left(x^{\prime}, x_{n}\right) \in \mathbb{R}^{n} \mid x_{n}>0\right\}$. Set

$$
\mathcal{E}_{v, \epsilon, t} \equiv\left\{\begin{array}{ll}
1 & \text { in } \Omega_{f}^{\epsilon} / t \\
v & \text { in } \Omega_{m}^{\epsilon} / t
\end{array} \quad \text { for } t \in(0,1]\right.
$$

Lemma 4.5 For any $\delta, \mathbf{M}>0$, there are $\tilde{\theta}_{1}, \tilde{\theta}_{2} \in(0,1)$ (depending on $\delta, \mathbf{M}, \mathbf{K}, Y_{f}, \Omega$ ) satisfying $\tilde{\theta}_{1}<\tilde{\theta}_{2}^{2}$ and there is a $\tilde{\omega}_{0}>0$ (depending on $\tilde{\theta}_{1}, \tilde{\theta}_{2}, \delta, \mathbf{M}, \mathbf{K}, \Omega$ ) satisfying $\tilde{\omega}_{0}<\omega_{0}\left(\omega_{0}\right.$ is that in Lemma 4.1$)$ such that if

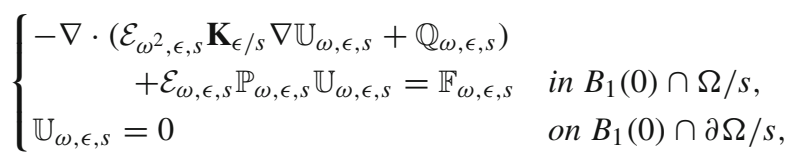

and if

$$
\left\{\begin{array}{c}
\omega, \frac{\epsilon}{s} \in\left(0, \tilde{\omega}_{0}\right), s \in(0,1], \tilde{\theta} \in\left[\tilde{\theta}_{1}, \tilde{\theta}_{2}\right], \mathbb{P}_{\omega, \epsilon, s}(x) \in[0, \mathbf{M}] \text { for } x \in B_{1}(0) \cap \Omega / s, \\
\max \left\{\left\|\mathcal{E}_{\omega, \epsilon, s} \mathbb{U}_{\omega, \epsilon, s}\right\|_{L^{2}\left(B_{1}(0) \cap \Omega / s\right)},\left\|\frac{1}{\tilde{\omega}_{0}} \mathbb{Q}_{\omega, \epsilon, s} \mathcal{X}_{\Omega_{f}^{\epsilon} / s}+\frac{1}{\omega} \mathbb{Q}_{\omega, \epsilon, s} \mathcal{X}_{\Omega_{m}^{\epsilon} / s}\right\|_{L^{n+\delta}\left(B_{1}(0)\right)},\right. \\
\left.\frac{1}{\tilde{\omega}_{0}}\left\|\mathbb{F}_{\omega, \epsilon, s} \mathcal{X}_{\Omega_{f}^{\epsilon} / s}+\frac{1}{\omega} \max \left\{\omega, \frac{\epsilon}{s}\right\} \mathbb{F}_{\omega, \epsilon, s} \mathcal{X}_{\Omega_{m}^{\epsilon} / s}\right\|_{L^{n+\delta}\left(B_{1}(0)\right)}\right\} \leq 1,
\end{array}\right.
$$


then

$$
\left\{\begin{array}{l}
f_{B_{\tilde{\theta}}(0) \cap \Omega / s}\left|\Pi_{\epsilon / s} \mathbb{U}_{\omega, \epsilon, s}\right| \Omega_{f}^{\epsilon} /\left.s\right|^{2} d x \leq \tilde{\theta}^{2 \mu} \\
f_{B_{\tilde{\theta}}(0) \cap \Omega_{m}^{\epsilon} / s} \omega^{2}\left|\mathbb{U}_{\omega, \epsilon, s}\right|^{2} d x \leq \tilde{\theta}^{2 \mu}
\end{array}\right.
$$

where $\mu \equiv \frac{\delta}{n+\delta}$.

Proof Consider the following problem

$$
\begin{cases}-\nabla \cdot\left(\mathcal{K}_{0} \nabla \mathbb{U}\right)+\mathbb{P U}=0 & \text { in } B_{3 / 4}(0) \cap \Omega / t, \\ \mathbb{U}=0 & \text { on } B_{3 / 4}(0) \cap \partial \Omega / t,\end{cases}
$$

where $t \in[0,1], \mathbb{P}(x) \in[0, \mathbf{M}]$ for $x \in B_{3 / 4}(0) \cap \Omega / t$, and $\mathcal{K}_{0}$ is defined in (2.9). Any solution $\mathbb{U}$ of (4.22) satisfies, by Theorem 9.13 [16] and (3.19),

$$
\|\mathbb{U}\|_{C^{1, \alpha}\left(\overline{B_{1 / 2}(0) \cap \Omega / t}\right)} \leq c\|\mathbb{U}\|_{L^{2}\left(B_{3 / 4}(0) \cap \Omega / t\right)},
$$

where $\alpha \in(0,1)$ and $c$ is a constant depending on $\mathbf{M}, \mathcal{K}_{0}, \Omega$ but independent of $t$. If $\check{\mu}$ satisfies $\mu<\check{\mu}<1$, by (4.23),

$$
f_{B_{\tilde{\theta}}(0) \cap \Omega / t} \mathbb{U}^{2} \mathrm{~d} x \leq \tilde{\theta}^{2 \check{\mu}} f_{B_{3 / 4}(0) \cap \Omega / t} \mathbb{U}^{2} \mathrm{~d} x
$$

for small $\tilde{\theta}$ (depending on $\delta, \mathbf{M}, \mathcal{K}_{0}, \Omega$ ). Fix $\tilde{\theta}_{1}, \tilde{\theta}_{2} \in\left(0, \frac{1}{2}\right)$ such that $\tilde{\theta}_{1}<\tilde{\theta}_{2}^{2}$ and (4.24) holds for any $\tilde{\theta} \in\left[\tilde{\theta}_{1}, \tilde{\theta}_{2}\right]$.

We claim (4.21)1. If not, there is a sequence $\left\{s_{\omega, \epsilon}, \tilde{\theta}_{\omega, \epsilon}, \mathbb{P}_{\omega, \epsilon, s_{\omega, \epsilon}}, \mathbb{U}_{\omega, \epsilon, s_{\omega, \epsilon}}, \mathbb{Q}_{\omega, \epsilon, s_{\omega, \epsilon}}\right.$, $\left.\mathbb{F}_{\omega, \epsilon, s_{\omega, \epsilon}}\right\}$ satisfying (4.20) and, as $\omega, \epsilon \rightarrow 0$,

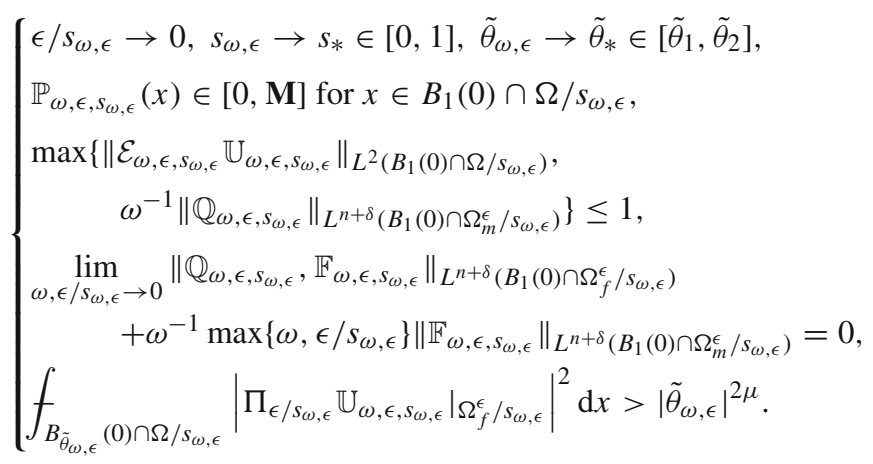

By energy method and A2, there is a constant $c$ independent of $\omega, \epsilon, s_{\omega, \epsilon}$ such that

$$
\begin{aligned}
& \left\|\mathbb{U}_{\omega, \epsilon, s_{\omega, \epsilon}}\right\|_{H^{1}\left(B_{4 / 5}(0) \cap \Omega_{f}^{\epsilon} / s_{\omega, \epsilon}\right)} \\
& \quad+\left\|\omega \nabla \mathbb{U}_{\omega, \epsilon, s_{\omega, \epsilon}}, \omega^{1 / 2} \mathbb{P}_{\omega, \epsilon, s_{\omega, \epsilon}}^{1 / 2} \mathbb{U}_{\omega, \epsilon, s_{\omega, \epsilon}}\right\|_{L^{2}\left(B_{4 / 5}(0) \cap \Omega_{m}^{\epsilon} / s_{\omega, \epsilon}\right)} \leq c .
\end{aligned}
$$

By compactness principle and by tracing the proof of Theorem 2.3 [3], we can extract a subsequence (same notation for subsequence) such that, as $\omega, \epsilon / s_{\omega, \epsilon} \rightarrow 0$,

$$
\begin{cases}\left.\Pi_{\epsilon / s_{\omega, \epsilon}} \mathbb{U}_{\omega, \epsilon, s_{\omega, \epsilon}}\right|_{\Omega_{f}^{\epsilon} / s_{\omega, \epsilon} \rightarrow \mathbb{U}} & \text { in } L^{2}\left(B_{3 / 4}(0) \cap \Omega / s_{*}\right) \text { strongly, } \\ \mathcal{E}_{\omega^{2}, \epsilon, s_{\omega, \epsilon}} \mathbf{K}_{\epsilon / s_{\omega, \epsilon}} \nabla \mathbb{U}_{\omega, \epsilon, s_{\omega, \epsilon}} \rightarrow \mathcal{K}_{0} \nabla \mathbb{U} & \text { in } L^{2}\left(B_{3 / 4}(0) \cap \Omega / s_{*}\right) \text { weakly, } \\ \mathcal{E}_{\omega, \epsilon, s_{\omega, \epsilon}} \mathbb{P}_{\omega, \epsilon, s_{\omega, \epsilon}} \mathbb{U}_{\omega, \epsilon, s_{\omega, \epsilon}} \rightarrow \mathbb{P} & \text { in } L^{2}\left(B_{3 / 4}(0) \cap \Omega / s_{*}\right) \text { weakly, }\end{cases}
$$


where $\mathcal{K}_{0}$ is a constant symmetric positive definite matrix, $\ell_{5} \leq \mathcal{K}_{0} \leq \ell_{6}$ (see (2.9) and (3.19)), and $\mathbb{P}(x) \in[0, \mathbf{M}]$ for $x \in B_{3 / 4}(0) \cap \Omega / s_{*}$. In (4.26), function $\mathbb{U}$ satisfies (4.22) with $t=s_{*}$. By (4.24)-(4.25), we conclude

$$
\begin{aligned}
\left|\tilde{\theta}_{*}\right|^{2 \mu} & \leq \lim _{\omega, \epsilon / s_{\omega, \epsilon} \rightarrow 0} f_{B_{\tilde{\theta}_{\omega, \epsilon}}(0) \cap \Omega / s_{\omega, \epsilon}}\left|\Pi_{\epsilon / s_{\omega, \epsilon}} \mathbb{U}_{\omega, \epsilon, s_{\omega, \epsilon}}\right| \Omega_{f}^{\epsilon} /\left.s_{\omega, \epsilon}\right|^{2} \mathrm{~d} x \\
& =f_{B_{\tilde{\theta}_{*}}(0) \cap \Omega / s_{*}} \mathbb{U}^{2} \mathrm{~d} x \leq\left|\tilde{\theta}_{*}\right|^{2 \check{\mu}} f_{B_{3 / 4}(0) \cap \Omega / s_{*}} \mathbb{U}^{2} \mathrm{~d} x .
\end{aligned}
$$

But (4.27) is impossible if we take $\tilde{\theta}_{2}$ small enough. Therefore, there is a $\tilde{\omega}_{0}$ such that (4.21) holds for $\omega, \epsilon / s \leq \tilde{\omega}_{0}$. Clearly, $\tilde{\omega}_{0}$ can be chosen so that $\tilde{\omega}_{0}<\omega_{0}$ (see Lemma 4.1 for $\left.\omega_{0}\right)$. The proof of $(4.21)_{2}$ is similar to that of $(4.3)_{2}$, so we skip it.

Lemma 4.6 For any $\delta, \mathbf{M}>0$, there are $\tilde{\theta}_{1}, \tilde{\theta}_{2} \in(0,1)$ (depending on $\delta, \mathbf{M}, \mathbf{K}, Y_{f}, \Omega$ ) satisfying $\tilde{\theta}_{1}<\tilde{\theta}_{2}^{2}$ and there is a $\tilde{\omega}_{0}>0$ (depending on $\tilde{\theta}_{1}, \tilde{\theta}_{2}, \delta, \mathbf{M}, \mathbf{K}, \Omega$ ) satisfying $\tilde{\omega}_{0}<\omega_{0}\left(\omega_{0}\right.$ is that in Lemma 4.2) such that if

$$
\begin{cases}-\nabla \cdot\left(\mathbf{E}_{\omega^{2}, \epsilon} \mathbf{K}_{\epsilon} \nabla U_{\omega, \epsilon}+Q_{\omega, \epsilon}\right)+\mathbf{E}_{\omega, \epsilon} \mathbf{P}_{\omega, \epsilon} U_{\omega, \epsilon}=F_{\omega, \epsilon} & \text { in } B_{1}(0) \cap \Omega \\ U_{\omega, \epsilon}=0 & \text { on } B_{1}(0) \cap \partial \Omega\end{cases}
$$

and if $\omega, \epsilon \in\left(0, \tilde{\omega}_{0}\right), \tilde{\theta} \in\left[\tilde{\theta}_{1}, \tilde{\theta}_{2}\right], \mathbf{P}_{\omega, \epsilon}(x) \in[0, \mathbf{M}]$ for $x \in B_{1}(0) \cap \Omega$, and $k$ satisfying $\epsilon / \tilde{\theta}^{k} \leq \tilde{\omega}_{0}$, then

$$
\left\{\begin{array}{l}
\left.f_{B_{\tilde{\theta} k}(0) \cap \Omega}\left|\Pi_{\epsilon} U_{\omega, \epsilon}\right| \Omega_{f}^{\epsilon}\right|^{2} d x \leq \tilde{\theta}^{2 k \mu}\left|\tilde{J}_{\omega, \epsilon}\right|^{2} \\
f_{B_{\tilde{\theta}^{k}}(0) \cap \Omega_{m}^{\epsilon}} \omega^{2}\left|U_{\omega, \epsilon}\right|^{2} d x \leq \tilde{\theta}^{2 k \mu}\left|\tilde{J}_{\omega, \epsilon}\right|^{2}
\end{array}\right.
$$

where $\tilde{J}_{\omega, \epsilon} \equiv\left\|\mathbf{E}_{\omega, \epsilon} U_{\omega, \epsilon}\right\|_{L^{2}\left(B_{1}(0) \cap \Omega\right)}+\omega^{-1}\left\|Q_{\omega, \epsilon}, \frac{1}{\tilde{\omega}_{0}} \max \{\omega, \epsilon\} F_{\omega, \epsilon}\right\|_{L^{n+\delta}\left(B_{1}(0) \cap \Omega_{m}^{\epsilon}\right)}+$ $\frac{1}{\tilde{\omega}_{0}}\left\|Q_{\omega, \epsilon}, F_{\omega, \epsilon}\right\|_{L^{n+\delta}\left(B_{1}(0) \cap \Omega_{f}^{\epsilon}\right)}$ and $\mu \equiv \frac{\delta}{n+\delta}$.

Proof The proof is similar to that of Lemma 4.2 and is done by induction on $k$. For $k=1$, (4.29) is deduced from Lemma 4.5 with $s=1$. Suppose (4.29) holds for some $k$ with $\epsilon / \tilde{\theta}^{k} \leq \tilde{\omega}_{0}$, we define

$$
\left\{\begin{array}{l}
\mathbb{U}_{\omega, \epsilon, \tilde{\theta}^{k}}(x) \equiv \tilde{J}_{\omega, \epsilon}^{-1} \tilde{\theta}^{-k \mu} U_{\omega, \epsilon}\left(\tilde{\theta}^{k} x\right) \\
\mathbb{Q}_{\omega, \epsilon, \tilde{\theta}^{k}}(x) \equiv \tilde{J}_{\omega, \epsilon}^{-1} \tilde{\theta}^{k(1-\mu)} Q_{\omega, \epsilon}\left(\tilde{\theta}^{k} x\right) \\
\mathbb{F}_{\omega, \epsilon, \tilde{\theta}^{k}}(x) \equiv \tilde{J}_{\omega, \epsilon}^{-1} \tilde{\theta}^{k(2-\mu)} F_{\omega, \epsilon}\left(\tilde{\theta}^{k} x\right) \\
\mathbb{P}_{\omega, \epsilon, \tilde{\theta}^{k}}(x) \equiv \tilde{\theta}^{2 k} \mathbf{P}_{\omega, \epsilon}\left(\tilde{\theta}^{k} x\right)
\end{array}\right.
$$

Then they satisfy

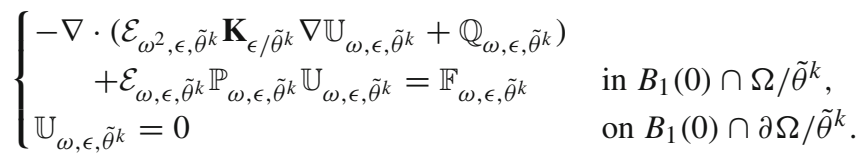

Following the argument of Lemma 4.2 and employing Lemma 4.5 with $s=\tilde{\theta}^{k}$, we obtain (4.29) with $k+1$ in place of $k$. 
Lemma 4.7 For any $\delta \in(0,3)$ and $\mathbf{M}>0$, there is a $\tilde{\omega}_{*} \in(0,1)$ (depending on $\left.\delta, \mathbf{M}, \mathbf{K}, Y_{f}, \Omega\right)$ such that if $\omega, \epsilon \leq \tilde{\omega}_{*}$ and $\mathbf{P}_{\omega, \epsilon}(x) \in[0, \mathbf{M}]$ for $x \in B_{1}(0) \cap \Omega$, then any solution of (4.28) satisfies

$$
\left[U_{\omega, \epsilon}\right]_{C^{0, \mu}\left(\overline{B_{1 / 2}(0) \cap \Omega_{f}^{\epsilon}}\right)}+\sup _{\substack{j \in \mathbb{Z}^{n} \\ \epsilon\left(Y_{m}+j\right) \subset B_{1 / 2}(0) \cap \Omega_{m}^{\epsilon}}} \omega^{3 / 2}\left[U_{\omega, \epsilon}\right]_{C^{0, \mu}\left(\epsilon\left(\overline{Y_{m}}+j\right)\right)} \leq c \hat{J}_{\omega, \epsilon},
$$

where $\mu \equiv \frac{\delta}{n+\delta} ; c$ is a constant independent of $\omega, \epsilon ; \hat{J}_{\omega, \epsilon}$ is defined as

$$
\begin{aligned}
\hat{J}_{\omega, \epsilon} \equiv & \frac{3}{\tilde{\omega}_{*}}\left(\left\|\mathbf{E}_{\omega, \epsilon} U_{\omega, \epsilon}\right\|_{L^{2}\left(B_{1}(0) \cap \Omega\right)}+\left\|Q_{\omega, \epsilon}, F_{\omega, \epsilon}\right\|_{L^{n+\delta}\left(B_{1}(0) \cap \Omega_{f}^{\epsilon}\right)}\right. \\
& \left.+\omega^{-1}\left\|Q_{\omega, \epsilon}, \max \{\omega, \epsilon\} F_{\omega, \epsilon}\right\|_{L^{n+\delta}\left(B_{1}(0) \cap \Omega_{m}^{\epsilon}\right)}\right) .
\end{aligned}
$$

Proof Let $\tilde{\theta}_{1}, \tilde{\theta}_{2}, \tilde{\omega}_{0}, \tilde{J}_{\omega, \epsilon}$ be same as those in Lemma 4.6, set $\tilde{\omega}_{*} \equiv \min \left\{\tilde{\omega}_{0} \tilde{\theta}_{2} / 3, \omega_{*}\right\}$ where $\omega_{*}$ is the one in Lemma 4.3, and let $\omega, \epsilon \leq \tilde{\omega}_{*}$. Denote by $c$ a constant independent of $\omega, \epsilon$. By energy method and A2, any solution of (4.28) satisfies

$$
\begin{aligned}
\left\|U_{\omega, \epsilon}\right\|_{H^{1}\left(B_{3 / 4}(0) \cap \Omega_{f}^{\epsilon}\right)} \leq & c\left(\left\|\mathbf{E}_{\omega, \epsilon} U_{\omega, \epsilon}\right\|_{L^{2}\left(B_{1}(0) \cap \Omega\right)}+\left\|\mathbf{E}_{1 / \omega, \epsilon} Q_{\omega, \epsilon}\right\|_{L^{2}\left(B_{1}(0) \cap \Omega\right)}\right. \\
& \left.+\left\|F_{\omega, \epsilon}\right\|_{L^{2}\left(B_{1}(0) \cap \Omega\right)}+\epsilon \omega^{-1}\left\|F_{\omega, \epsilon}\right\|_{L^{2}\left(B_{1}(0) \cap \Omega_{m}^{\epsilon}\right)}\right) \equiv c \check{\mathcal{I}} .
\end{aligned}
$$

By Theorem 7.26 [16] and Remark 2.1,

$$
\left\|\left.\Pi_{\epsilon} U_{\omega, \epsilon}\right|_{\Omega_{f}^{\epsilon}}\right\|_{L^{\frac{2 n}{n-2}\left(B_{3 / 4}(0) \cap \Omega\right)}} \leq c \check{\mathcal{I}} .
$$

For any $x \in B_{\tilde{\theta}_{2} / 3}(0) \cap \Omega_{f}^{\epsilon}$, define $\eta(x) \equiv\left|x-x_{0}\right|$ where $x_{0} \in \partial \Omega$ satisfying $\left|x-x_{0}\right|=$ $\min _{y \in \partial \Omega}|x-y|$. Then we have either case (1) $\eta(x)>\frac{2 \epsilon}{3 \tilde{\omega}_{0}}$ or case (2) $\eta(x) \leq \frac{2 \epsilon}{3 \tilde{\omega}_{0}}$.

Let us consider case (1). Because of $\tilde{\theta}_{1}<\tilde{\theta}_{2}^{2}$, for any $r \in\left[\epsilon / \tilde{\omega}_{0}, \tilde{\theta}_{2}\right]$, there are $\tilde{\theta} \in\left[\tilde{\theta}_{1}, \tilde{\theta}_{2}\right]$ and $k \in \mathbb{N}$ satisfying $r=\tilde{\theta}^{k}$. Since $\eta(x) \in\left[\frac{2 \epsilon}{3 \tilde{\omega}_{0}}, \frac{\tilde{\theta}_{2}}{3}\right]$, by Lemma 4.6,

$$
\left\{\begin{array}{l}
\left.f_{B_{r}\left(x_{0}\right) \cap \Omega}\left|\Pi_{\epsilon} U_{\omega, \epsilon}\right| \Omega_{f}^{\epsilon}\right|^{2} \mathrm{~d} y \leq r^{2 \mu}\left|\tilde{J}_{\omega, \epsilon}\right|^{2} \\
f_{B_{r}\left(x_{0}\right) \cap \Omega_{m}^{\epsilon}} \omega^{2}\left|U_{\omega, \epsilon}\right|^{2} \mathrm{~d} y \leq r^{2 \mu}\left|\tilde{J}_{\omega, \epsilon}\right|^{2}
\end{array} \quad \text { for } r \in\left[\frac{3}{2} \eta(x), \tilde{\theta}_{2}\right] .\right.
$$

So, for $s \in\left[\frac{\eta(x)}{2}, \frac{\tilde{\theta}_{2}}{3}\right]$,

$$
\left\{\begin{array}{l}
f_{B_{s}(x) \cap \Omega}\left|\Pi_{\epsilon} U_{\omega, \epsilon}\right|_{\Omega_{f}^{\epsilon}}-\left.\left(\left.\Pi_{\epsilon} U_{\omega, \epsilon}\right|_{\Omega_{f}^{\epsilon}}\right)_{x, s}\right|^{2} \mathrm{~d} y \leq c s^{2 \mu}\left|\tilde{J}_{\omega, \epsilon}\right|^{2}, \\
f_{B_{s}(x) \cap \Omega_{m}^{\epsilon}} \omega^{2}\left|U_{\omega, \epsilon}-\left(\left.\Pi_{\epsilon} U_{\omega, \epsilon}\right|_{\Omega_{f}^{\epsilon}}\right)_{x, s}\right|^{2} \mathrm{~d} y \leq c s^{2 \mu}\left|\tilde{J}_{\omega, \epsilon}\right|^{2} .
\end{array}\right.
$$

Next we shift the coordinate system such that $x$ is located at the origin and we define, in $B_{1}(x) \backslash \partial \Omega_{m}^{\epsilon} / \eta(x)$,

$$
\left\{\begin{array}{l}
\mathbb{U}_{\omega, \epsilon, \eta(x)}(y) \equiv \frac{U_{\omega, \epsilon}(\eta(x) y)-\left(\left.\Pi_{\epsilon} U_{\omega, \epsilon}\right|_{\Omega_{f}^{\epsilon}}\right)_{x, \eta(x)}}{\hat{J}_{\omega, \epsilon} \eta^{\mu}(x)} \\
\mathbb{Q}_{\omega, \epsilon, \eta(x)}(y) \equiv \frac{Q_{\omega, \epsilon}(\eta(x) y)}{\hat{J}_{\omega, \epsilon} \eta^{\mu-1}(x)}, \\
\mathbb{F}_{\omega, \epsilon, \eta(x)}(y) \equiv \frac{F_{\omega, \epsilon}(\eta(x) y)-\mathbf{E}_{\omega, \epsilon / \eta(x)}(y) \mathbf{P}_{\omega, \epsilon}(\eta(x) y)\left(\left.\Pi_{\epsilon} U_{\omega, \epsilon}\right|_{\Omega_{f}^{\epsilon}}\right)_{x, \eta(x)}}{\hat{J}_{\omega, \epsilon} \eta^{\mu-2}(x)} \\
\mathbb{P}_{\omega, \epsilon, \eta(x)}(y) \equiv \mathbf{P}_{\omega, \epsilon}(\eta(x) y) .
\end{array}\right.
$$


See (4.31) for $\hat{J}_{\omega, \epsilon}$. Then these functions satisfy

$$
\begin{aligned}
-\nabla \cdot & \left(\mathbf{E}_{\omega^{2}, \epsilon / \eta(x)} \mathbf{K}_{\epsilon / \eta(x)} \nabla \mathbb{U}_{\omega, \epsilon, \eta(x)}+\mathbb{Q}_{\omega, \epsilon, \eta(x)}\right) \\
& +|\eta(x)|^{2} \mathbf{E}_{\omega, \epsilon / \eta(x)} \mathbb{P}_{\omega, \epsilon, \eta(x)} \mathbb{U}_{\omega, \epsilon, \eta(x)}=\mathbb{F}_{\omega, \epsilon, \eta(x)} \quad \text { in } B_{1}(x) .
\end{aligned}
$$

Take $s=\eta(x)<1$ in (4.33) to see, by (4.32),

$$
\left\{\begin{array}{l}
\mathbb{P}_{\omega, \epsilon, \eta(x)}(y) \in[0, \mathbf{M}] \text { for all } y \in B_{1}(x) . \\
\tilde{\omega}_{0}^{-1}\left(\left\|\mathbf{E}_{\omega, \epsilon / \eta(x)} \mathbb{U}_{\omega, \epsilon, \eta(x)}\right\|_{L^{2}\left(B_{1}(x)\right)}+\left\|\mathbb{Q}_{\omega, \epsilon, \eta(x)}, \mathbb{F}_{\omega, \epsilon, \eta(x)}\right\|_{L^{n+\delta}\left(B_{1}(x) \cap \Omega_{f}^{\epsilon} / \eta(x)\right)}\right. \\
\left.\quad+\omega^{-1}\left\|\mathbb{Q} \omega, \epsilon, \eta(x), \max \{\omega, \epsilon / \eta(x)\} \mathbb{F}_{\omega, \epsilon, \eta(x)}\right\|_{L^{n+\delta}\left(B_{1}(x) \cap \Omega_{m}^{\epsilon} / \eta(x)\right)}\right) \leq c .
\end{array}\right.
$$

Apply Lemma 4.3 to (4.34) to obtain

$$
+\left[\mathbb{U}_{\omega, \epsilon, \eta(x)}\right]_{C^{0, \mu}\left(\overline{B_{1 / 2}(x) \cap \Omega_{f}^{\epsilon} / \eta(x)}\right)}+\sup _{\substack{j \in \mathbb{Z}^{n} \\ \eta(x)}} \omega_{m}^{3 / 2}\left[\mathbb{U}_{\omega, \epsilon, \eta(x)}\right]_{C^{0, \mu}\left(\frac{\epsilon}{\eta(x)}\left(\overline{Y_{m}}+j\right)\right)} \leq c .
$$

Which implies

$$
f_{B_{s}(x) \cap \Omega}\left|\Pi_{\epsilon} U_{\omega, \epsilon}\right|_{\Omega_{f}^{\epsilon}}-\left.\left(\Pi_{\epsilon} U_{\omega, \epsilon} \mid \Omega_{f}^{\epsilon}\right)_{x, s}\right|^{2} \mathrm{~d} y \leq c s^{2 \mu}\left|\hat{J}_{\omega, \epsilon}\right|^{2} \quad \text { for } s<\frac{\eta(x)}{2} .
$$

Next we consider case (2). Because of $\tilde{\theta}_{1}<\tilde{\theta}_{2}^{2}$, for any $r \in\left[\epsilon / \tilde{\omega}_{0}, \tilde{\theta}_{2}\right]$, there are $\tilde{\theta} \in$ $\left[\tilde{\theta}_{1}, \tilde{\theta}_{2}\right]$ and $k \in \mathbb{N}$ satisfying $r=\tilde{\theta}^{k}$. By Lemma 4.6,

$$
\left\{\begin{array}{l}
\left.f_{B_{r}\left(x_{0}\right) \cap \Omega}\left|\Pi_{\epsilon} U_{\omega, \epsilon}\right| \Omega_{f}^{\epsilon}\right|^{2} \mathrm{~d} y \leq c r^{2 \mu}\left|\tilde{J}_{\omega, \epsilon}\right|^{2} \\
f_{B_{r}\left(x_{0}\right) \cap \Omega_{m}^{\epsilon}} \omega^{2}\left|U_{\omega, \epsilon}\right|^{2} \mathrm{~d} y \leq c r^{2 \mu}\left|\tilde{J}_{\omega, \epsilon}\right|^{2}
\end{array} \text { for } r \in\left[\epsilon / \tilde{\omega}_{0}, \tilde{\theta}_{2}\right] .\right.
$$

This implies, for $s \in\left[\frac{\epsilon}{3 \tilde{\omega}_{0}}, \frac{\tilde{\theta}_{2}}{3}\right]$,

$$
\left\{\begin{array}{l}
f_{B_{s}(x) \cap \Omega}\left|\Pi_{\epsilon} U_{\omega, \epsilon}\right| \Omega_{f}^{\epsilon}-\left.\left(\Pi_{\epsilon} U_{\omega, \epsilon} \mid \Omega_{f}^{\epsilon}\right)_{x, s}\right|^{2} \mathrm{~d} y \leq c s^{2 \mu}\left|\tilde{J}_{\omega, \epsilon}\right|^{2}, \\
f_{B_{s}(x) \cap \Omega_{m}^{\epsilon}} \omega^{2}\left|U_{\omega, \epsilon}-\left(\Pi_{\epsilon} U_{\omega, \epsilon} \mid \Omega_{f}^{\epsilon}\right)_{x, s}\right|^{2} \mathrm{~d} y \leq c s^{2 \mu}\left|\tilde{J}_{\omega, \epsilon}\right|^{2} .
\end{array}\right.
$$

Again we shift the coordinate system such that $x$ is located at the origin. Define, in $\left(B_{1 / \tilde{\omega}_{0}}(x) \cap\right.$ $\Omega / \epsilon) \backslash \partial \Omega_{m}^{\epsilon} / \epsilon$,

$$
\left\{\begin{array}{l}
\mathbb{U}_{\omega, 1}(y) \equiv \hat{J}_{\omega, \epsilon}^{-1} \epsilon^{-\mu}\left(U_{\omega, \epsilon}(\epsilon y)-\left(\left.\Pi_{\epsilon} U_{\omega, \epsilon}\right|_{\Omega_{f}^{\epsilon}}\right)_{x, \epsilon / \tilde{\omega}_{0}}\right), \\
\mathbb{Q}_{\omega, 1}(y) \equiv \hat{J}_{\omega, \epsilon}^{-1} \epsilon^{1-\mu} Q_{\omega, \epsilon}(\epsilon y), \\
\mathbb{F}_{\omega, 1}(y) \equiv \hat{J}_{\omega, \epsilon}^{-1} \epsilon^{2-\mu}\left(F_{\omega, \epsilon}(\epsilon y)-\mathcal{E}_{\omega, \epsilon, \epsilon}(y) \mathbf{P}_{\omega, \epsilon}(\epsilon y)\left(\left.\Pi_{\epsilon} U_{\omega, \epsilon}\right|_{\Omega_{f}^{\epsilon}}\right)_{x, \epsilon / \tilde{\omega}_{0}}\right), \\
\mathbb{P}_{\omega, 1}(y) \equiv \epsilon^{2} \mathbf{P}_{\omega, \epsilon}(\epsilon y),
\end{array}\right.
$$

and define

$$
\mathbb{U}_{b_{\omega}} \equiv-\hat{J}_{\omega, \epsilon}^{-1} \epsilon^{-\mu}\left(\left.\Pi_{\epsilon} U_{\omega, \epsilon}\right|_{\Omega_{f}^{\epsilon}}\right)_{x, \epsilon / \tilde{\omega}_{0}} \quad \text { in } B_{\tilde{\omega}_{0}}(x) \cap \Omega / \epsilon .
$$

See (4.19) for $\mathcal{E}_{\omega, \epsilon, \epsilon}$. 
By $(4.37)_{1}, \mathbb{U}_{b_{\omega}}$ is a constant independent of $\omega, \epsilon$. Then these functions satisfy

$$
\begin{cases}-\nabla \cdot\left(\mathcal{E}_{\omega^{2}, \epsilon, \epsilon} \mathbf{K} \nabla \mathbb{U}_{\omega, 1}+\mathbb{Q} \omega, 1\right)+\mathcal{E}_{\omega, \epsilon, \epsilon} \mathbb{P}_{\omega, 1} \mathbb{U}_{\omega, 1}=\mathbb{F}_{\omega, 1} & \text { in } B_{\frac{1}{\tilde{\omega}_{0}}}(x) \cap \Omega / \epsilon, \\ \mathbb{U}_{\omega, 1}=\mathbb{U}_{b_{\omega}} & \text { on } B_{\frac{1}{\tilde{\omega}_{0}}}(x) \cap \partial \Omega / \epsilon .\end{cases}
$$

Take $s=\frac{\epsilon}{\tilde{\omega}_{0}}$ in (4.38) to see, by $(4.37)_{1}$,

$$
\left\{\begin{array}{l}
\mathbb{P}_{\omega, 1} \in[0, \mathbf{M}] \text { for all } x \in B_{1 / \tilde{\omega}_{0}}(x) \cap \Omega / \epsilon, \\
\left\|\mathcal{E}_{\omega, \epsilon, \epsilon} \mathbb{U}_{\omega, 1}\right\|_{L^{2}\left(B_{1 / \tilde{\omega}_{0}}(x) \cap \Omega / \epsilon\right)}+\left\|\mathcal{E}_{1 / \omega, \epsilon, \epsilon} \mathbb{Q} \omega, 1, \mathcal{E}_{1 / \omega, \epsilon, \epsilon} \mathbb{F}_{\omega, 1}\right\|_{L^{n+\delta}\left(B_{1 / \tilde{\omega}_{0}}(x) \cap \Omega / \epsilon\right)} \\
\quad+\left\|\mathbb{U}_{b_{\omega}}\right\|_{W^{1, n+\delta}\left(B_{1 / \tilde{\omega}_{0}}(x) \cap \Omega / \epsilon\right)} \leq c .
\end{array}\right.
$$

By Lemma 3.4,

$$
\left[\mathbb{U}_{\omega, 1}\right]_{C^{0, \mu}\left(\overline{B_{1 / 2 \tilde{\omega}_{0}}(x) \cap \Omega_{f}^{\epsilon} / \epsilon}\right)}+\omega^{3 / 2}\left[\mathbb{U}_{\omega, 1}\right]_{C^{0, \mu}\left(\overline{B_{1 / 2 \tilde{\omega}_{0}}(x) \cap \Omega_{m}^{\epsilon} / \epsilon}\right)} \leq c .
$$

(4.39) imply (4.38) $)_{1}$ holds for $s \leq \frac{\epsilon}{2 \tilde{\omega}_{0}}$.

The Hölder estimate of $\Pi_{\epsilon} U_{\omega, \epsilon}$ follows from (4.33) 1 , (4.36), (4.38) 1 , (4.39), and Theorem 1.2 in page 70 [15]. The Hölder estimate of $U_{\omega, \epsilon}$ in $\epsilon\left(\overline{Y_{m}}+j\right) \subset B_{1 / 2}(0) \cap \overline{\Omega_{m}^{\epsilon}}$ is from (4.35) and (4.39).

Remark 4.2 Let $\tilde{\omega}_{*}$ be same as that in Lemma 4.7. By Lemma 3.4 with $p=n+\delta$, we know that if $\delta \in(0,3), \mathbf{M}>0, \epsilon \in\left[\tilde{\omega}_{*}, 1\right], \omega \in\left(0, \tilde{\omega}_{*}\right)$, and $\mathbf{P}_{\omega, \epsilon}(x) \in[0, \mathbf{M}]$ for all $x \in B_{1}(0) \cap \Omega$, any solution of (4.28) satisfies (4.30). Together with Lemma 4.7, any solution of (4.28) satisfies (4.30) if $\delta \in(0,3), \mathbf{M}>0, \epsilon \in(0,1), \omega \in\left(0, \tilde{\omega}_{*}\right)$, and $\mathbf{P}_{\omega, \epsilon}(x) \in[0, \mathbf{M}]$ for all $x \in B_{1}(0) \cap \Omega$.

Let us consider the solutions of (4.28) with $\mathbf{P}_{\omega, \epsilon}=0$. By tracing the arguments of Lemma 4.7 and Remark 4.2 and employing Lemma 3.4, then we have

Lemma 4.8 For any $\delta>0$, there is a $\tilde{\omega}_{*} \in(0,1)$ (depending on $\left.\delta, \mathbf{K}, Y_{f}, \Omega\right)$ such that, if $\omega \in\left(0, \tilde{\omega}_{*}\right)$ and $\epsilon \in(0,1)$, then any solution of (4.28) with $\mathbf{P}_{\omega, \epsilon}=0$ satisfies

$$
\left[U_{\omega, \epsilon}\right]_{C^{0, \mu}\left(\overline{B_{1 / 2}(0) \cap \Omega_{f}^{\epsilon}}\right)}+\sup _{\substack{j \in \mathbb{Z}^{n} \\ \epsilon\left(Y_{m}+j\right) \subset B_{1 / 2}(0) \cap \Omega_{m}^{\epsilon}}} \omega\left[U_{\omega, \epsilon}\right]_{C^{0, \mu}\left(\epsilon\left(\overline{Y_{m}}+j\right)\right)} \leq c \hat{J}_{\omega, \epsilon},
$$

where $c$ is a constant independent of $\omega, \epsilon$. See Lemma 4.7 for $\mu, \hat{J}_{\omega, \epsilon}$.

By energy method, partition of unity, Remark 4.1, Remark 4.2, Lemma 4.4, Lemma 4.8, and Poincaré inequality [16], we conclude

Lemma 4.9 Under $A 1-A 2$, for any $\delta \in(0,3)$ and $\mathbf{M}>0$, there is a constant $\tilde{\omega}_{*} \in(0,1)$ (depending on $\delta, \mathbf{M}, \mathbf{K}, Y_{f}, \Omega$ ) such that if

$$
\begin{cases}-\nabla \cdot\left(\mathbf{E}_{\omega^{2}, \epsilon} \mathbf{K}_{\epsilon} \nabla U_{\omega, \epsilon}+Q_{\omega, \epsilon}\right)+\mathbf{E}_{\omega, \epsilon} \mathbf{P}_{\epsilon} U_{\omega, \epsilon}=F_{\omega, \epsilon} & \text { in } \Omega, \\ U_{\omega, \epsilon}=0 & \text { on } \partial \Omega,\end{cases}
$$

and if $\omega \in\left(0, \tilde{\omega}_{*}\right), \epsilon \in(0,1)$, and $\mathbf{P}_{\omega, \epsilon}(x) \in[0, \mathbf{M}]$ for all $x \in \Omega$, then

$$
\begin{aligned}
& {\left[U_{\omega, \epsilon}\right]_{C^{0, \mu}\left(\overline{\Omega_{f}^{\epsilon}}\right)}+\sup _{\substack{j \in \mathbb{Z}^{n} \\
\epsilon\left(Y_{m}+j\right) \subset \Omega_{m}^{\epsilon}}} \omega^{\lambda}\left[U_{\omega, \epsilon}\right]_{C^{0, \mu}\left(\epsilon\left(\overline{Y_{m}}+j\right)\right)}} \\
& \leq c\left(\left\|Q_{\omega, \epsilon}, F_{\omega, \epsilon}\right\|_{L^{n+\delta}\left(\Omega_{f}^{\epsilon}\right)}+\omega^{-1}\left\|Q_{\omega, \epsilon}, \max \{\omega, \epsilon\} F_{\omega, \epsilon}\right\|_{L^{n+\delta}\left(\Omega_{m}^{\epsilon}\right)}\right),
\end{aligned}
$$


where $\mu \equiv \frac{\delta}{n+\delta}$ and $c$ is a positive constant independent of $\omega, \epsilon$. Here $\lambda$ is $\frac{3}{2}$ if $\mathbf{P}_{\omega, \epsilon} \neq 0$ and is 1 if $\mathbf{P}_{\omega, \epsilon}=0$.

Under A1-A4, we multiply (1.1) by $\left|\Psi_{\omega, \epsilon}\right|^{q-2} \Psi_{\omega, \epsilon}$ for $q>2$ and integrate over $\Omega$ to obtain

$$
\left\|\Psi_{\omega, \epsilon}\right\|_{L^{q}(\Omega)} \leq c\left\|\mathbf{E}_{1 / \omega, \epsilon} V_{\omega, \epsilon}, G_{\omega, \epsilon}\right\|_{L^{q}(\Omega)},
$$

where $c$ is independent of $\omega, \epsilon$. Then we write (1.1) as

$$
\begin{cases}-\nabla \cdot\left(\mathbf{E}_{\omega^{2}, \epsilon} \mathbf{K}_{\epsilon} \nabla \Psi_{\omega, \epsilon}+V_{\omega, \epsilon}\right)=G_{\omega, \epsilon}-\mathbf{T}_{\omega, \epsilon} \Psi_{\omega, \epsilon} & \text { in } \Omega \\ \Psi_{\omega, \epsilon}=0 & \text { on } \partial \Omega .\end{cases}
$$

Theorem 2.1 follows by energy method, (4.40) for $q=n+\delta$, and Lemma 4.9 for $\mathbf{P}_{\omega, \epsilon}=0$. Theorem 2.2 is a direct consequence of energy method and Lemma 4.9.

\section{Convergence estimates}

In this section, we prove Theorems 2.3, 2.4, 2.5. For each $v \in(0,1)$ and $i_{1}, i_{2} \in\{1,2, \cdots, n\}$, we find $\mathbb{Y}_{v}^{\left(i_{1}, i_{2}\right)} \in H_{p e r}^{1}\left(\mathbb{R}^{n}\right)$ satisfying

$$
\left\{\begin{array}{l}
\nabla \cdot\left(\mathbb{E}_{v^{2}} \mathbf{K}\left(\nabla \mathbb{Y}_{v}^{\left(i_{1}, i_{2}\right)}+\mathbb{X}_{v}^{\left(i_{2}\right)} \vec{e}_{i_{1}}\right)\right) \\
=\frac{\mathcal{K}_{v}^{\left(i_{1}, i_{2}\right)}}{\left|Y_{f}\right|} \mathcal{X}_{Y_{f}}-\mathbb{E}_{v^{2}} \mathbf{K}\left(\partial_{i_{1}} \mathbb{X}_{v}^{\left(i_{2}\right)}+\delta_{i_{1}, i_{2}}\right) \quad \text { in } Y, \\
\int_{Y_{f}} \mathbb{Y}_{v}^{\left(i_{1}, i_{2}\right)}(y) \mathrm{d} y=0,
\end{array}\right.
$$

where $\vec{e}_{i_{1}}$ is a unit vector in the $i_{1}$ th coordinate direction, $\delta_{i_{1}, i_{2}}$ is 1 if $i_{1}=i_{2}$ and is 0 if $i_{1} \neq i_{2}$, and $\mathcal{K}_{v}^{\left(i_{1}, i_{2}\right)}$ is the $\left(i_{1}, i_{2}\right)$ th component of $\mathcal{K}_{v}$. See (2.5) for $\mathbb{X}_{v}^{(i)}$ and (2.9) for $\mathcal{K}_{v}$. By Lax-Milgram Theorem [16], A1-A2, and (3.17), $\mathbb{Y}_{v}^{\left(i_{1}, i_{2}\right)}$ is uniquely solvable. By Lemma 3.3 ,

$$
\left\|\mathbb{Y}_{v}^{\left(i_{1}, i_{2}\right)}\right\|_{C^{2}\left(\overline{Y_{f}}\right) \cap C^{2}\left(\overline{Y_{m}}\right)} \leq c
$$

where $c$ is a constant independent of $v$. Define $n \times n$ matrices $\mathbb{Y}_{v} \equiv\left(\mathbb{Y}_{v}^{\left(i_{1}, i_{2}\right)}\right)$ and $\mathbb{Y}_{v, s}(x) \equiv$ $s^{2} \mathbb{Y}_{v}\left(\frac{x}{s}\right)$ for $v, s \in(0,1)$.

\subsection{Proof of Theorem 2.3}

A1-A6 are assumed. This subsection consists of two parts. The first part is for $\omega, \epsilon \rightarrow 0$, $\frac{\omega}{\epsilon} \rightarrow \infty$, and the second part is for $\omega, \epsilon \rightarrow 0, \frac{\omega}{\epsilon} \rightarrow \sigma \in[0, \infty)$.

\subsubsection{Part 1: $\omega, \epsilon \rightarrow 0, \frac{\omega}{\epsilon} \rightarrow \infty$.}

For each $v \in(0,1)$, we find $\widetilde{\mathbb{W}}_{v} \in H_{\text {per }}^{1}\left(\mathbb{R}^{n}\right)$ satisfying

$$
\left\{\begin{array}{l}
\nabla \cdot\left(\mathbb{E}_{v^{2}} \mathbf{K} \nabla \widetilde{\mathbb{W}}_{\nu}\right)=\mathbf{T}-\mathcal{T}_{\infty} \mathcal{X}_{Y_{f}} \quad \text { in } Y, \\
\int_{Y_{f}} \widetilde{\mathbb{W}}_{\nu}(y) \mathrm{d} y=0 .
\end{array}\right.
$$


See (2.11) for $\mathcal{T}_{\infty}$. By Lax-Milgram Theorem [16], $\widetilde{\mathbb{W}}_{v}$ is uniquely solvable. By Lemma 3.3 and A5,

$$
\left\|\mathbb{E}_{v} \widetilde{\mathbb{W}}_{v}\right\|_{C^{2}\left(\overline{Y_{f}}\right) \cap C^{2}\left(\overline{Y_{m}}\right)} \leq c / v
$$

where $c$ is independent of $\nu$. Define $\widetilde{\mathbb{W}}_{\nu, s}(x) \equiv s^{2} \widetilde{\mathbb{W}}_{\nu}\left(\frac{x}{s}\right)$ for $v, s \in(0,1)$.

Let $\Psi_{\omega, \epsilon}$ be the solution of (1.1) with $V_{\omega, \epsilon}=0, \Psi$ be the solution of (2.12), and

$$
\varphi_{\omega, \epsilon} \equiv \Psi_{\omega, \epsilon}-\Psi-\widetilde{\mathbb{W}}_{\omega, \epsilon} \Psi-\mathbb{X}_{\omega, \epsilon} \nabla \Psi-\mathbb{Y}_{\omega, \epsilon} \nabla^{2} \Psi \quad \text { in } \Omega .
$$

See (2.5) for $\mathbb{X}_{\omega, \epsilon}$ and (5.1) for $\mathbb{Y}_{\omega, \epsilon}$. By (2.12)-(2.13), (3.17), (3.19), and (5.1)-(5.4),

$$
\left\{\begin{array}{rlrl}
-\nabla & \cdot\left(\mathbf{E}_{\omega^{2}, \epsilon} \mathbf{K}_{\epsilon}\left(\nabla \varphi_{\omega, \epsilon}+\widetilde{\mathbb{W}}_{\omega, \epsilon} \nabla \Psi+\mathbb{Y}_{\omega, \epsilon} \nabla^{3} \Psi\right)\right)+\mathbf{T}_{\omega, \epsilon} \varphi_{\omega, \epsilon} & \\
\quad=\mathcal{O}_{1}(\omega+\epsilon / \omega)+G_{\omega, \epsilon}-\mathcal{G} \mathcal{X}_{\Omega_{f}^{\epsilon}} & & \text { in } \Omega \\
\varphi_{\omega, \epsilon} & =\mathcal{O}_{2}\left(\epsilon+\epsilon^{2} / \omega^{2}\right) & & \text { on } \partial \Omega
\end{array}\right.
$$

where $\mathcal{O}_{1}(v)$ denotes a function satisfying $\left\|\mathcal{O}_{1}(v)\right\|_{L^{n+\delta}(\Omega)} \leq c v$ and $\mathcal{O}_{2}(v)$ denotes a function satisfying $\left\|\mathcal{O}_{2}(v)\right\|_{L^{\infty}(\Omega)} \leq c v$ for some constant $c$ independent of $\omega$, $\epsilon$. See (2.12) for $\mathcal{G}$. Decompose $\varphi_{\omega, \epsilon}$ as $\varphi_{\omega, \epsilon}=\widehat{\varphi}_{\omega, \epsilon}+\check{\varphi}_{\omega, \epsilon}$, where $\widehat{\varphi}_{\omega, \epsilon}$ satisfies

$$
\left\{\begin{array}{rlrl}
-\nabla & \cdot\left(\mathbf{E}_{\omega^{2}, \epsilon} \mathbf{K}_{\epsilon}\left(\nabla \widehat{\varphi}_{\omega, \epsilon}+\widetilde{\mathbb{W}}_{\omega, \epsilon} \nabla \Psi+\mathbb{Y}_{\omega, \epsilon} \nabla^{3} \Psi\right)\right)+\mathbf{T}_{\omega, \epsilon} \widehat{\varphi}_{\omega, \epsilon} & \\
\quad=\mathcal{O}_{1}(\omega+\epsilon / \omega)+G_{\omega, \epsilon}-\mathcal{G} \mathcal{X}_{\Omega_{f}^{\epsilon}} & \text { in } \Omega, \\
\widehat{\varphi}_{\omega, \epsilon}=0 & \text { on } \partial \Omega,
\end{array}\right.
$$

and $\check{\varphi}_{\omega, \epsilon}$ satisfies

$$
\begin{cases}-\nabla \cdot\left(\mathbf{E}_{\omega^{2}, \epsilon} \mathbf{K}_{\epsilon} \nabla \check{\varphi}_{\omega, \epsilon}\right)+\mathbf{T}_{\omega, \epsilon} \check{\varphi}_{\omega, \epsilon}=0 & \text { in } \Omega, \\ \check{\varphi}_{\omega, \epsilon}=\mathcal{O}_{2}\left(\epsilon+\epsilon^{2} / \omega^{2}\right) & \text { on } \partial \Omega .\end{cases}
$$

By Theorem 2.1, A6, (2.13), (5.2), and (5.4), the solution of (5.5) satisfies

$$
\left\|\mathbf{E}_{\omega, \epsilon} \widehat{\varphi}_{\omega, \epsilon}\right\|_{L^{\infty}(\Omega)} \leq c\left(\max \{\omega, \epsilon / \omega\}+\left\|G_{\omega, \epsilon}-\mathcal{G}\right\|_{L^{n+\delta}\left(\Omega_{f}^{\epsilon}\right)}\right),
$$

where $c$ is independent of $\omega, \epsilon$. By Theorem 8.1 [16], the solution of (5.6) satisfies

$$
\left\|\check{\varphi}_{\omega, \epsilon}\right\|_{L^{\infty}(\Omega)}=\left\|\check{\varphi}_{\omega, \epsilon}\right\|_{L^{\infty}(\partial \Omega)} \leq c\left(\epsilon+\epsilon^{2} / \omega^{2}\right),
$$

where $c$ is a constant independent of $\omega, \epsilon$.

From (5.7) and (5.8), we see that the difference between the solution of (1.1) with $V_{\omega, \epsilon}=0$ and the solution of (2.12) satisfies

$$
\left\|\mathbf{E}_{\omega, \epsilon}\left(\Psi_{\omega, \epsilon}-\Psi\right)\right\|_{L^{\infty}(\Omega)} \leq c\left(\max \{\omega, \epsilon / \omega\}+\left\|G_{\omega, \epsilon}-\mathcal{G}\right\|_{L^{n+\delta}\left(\Omega_{f}^{\epsilon}\right)}\right),
$$

where $c$ is a constant independent of $\omega, \epsilon$. Now let us consider (2.14). We note that the solution of (1.1) with $V_{\omega, \epsilon}=0$ and the solution of (2.14) satisfy, for any $\epsilon\left(Y_{m}+j\right) \subset \Omega_{m}^{\epsilon}$ and $j \in \mathbb{Z}^{n}$,

$$
\begin{cases}-\omega^{2} \nabla \cdot\left(\mathbf{K}_{\epsilon} \nabla\left(\Psi_{\omega, \epsilon}-\phi_{\omega, \epsilon}^{(j)}\right)\right)+\mathbf{T}_{\omega, \epsilon}\left(\Psi_{\omega, \epsilon}-\phi_{\omega, \epsilon}^{(j)}\right)=0 & \text { in } \epsilon\left(Y_{m}+j\right) \\ \Psi_{\omega, \epsilon}-\phi_{\omega, \epsilon}^{(j)}=\Psi_{\omega, \epsilon}-\Psi & \text { on } \partial \epsilon\left(Y_{m}+j\right)\end{cases}
$$


By (5.9) and Theorem 8.1 [16], we conclude

$$
\left\|\Psi_{\omega, \epsilon}-\sum_{\substack{j \in \mathbb{Z}^{n} \\ \epsilon\left(Y_{m}+j\right) \subset \Omega_{m}^{\epsilon}}} \phi_{\omega, \epsilon}^{(j)}\right\|_{L^{\infty}\left(\Omega_{m}^{\epsilon}\right)} \leq c\left(\max \{\omega, \epsilon / \omega\}+\left\|G_{\omega, \epsilon}-\mathcal{G}\right\|_{L^{n+\delta}\left(\Omega_{f}^{\epsilon}\right)}\right),
$$

where $c$ is independent of $\omega, \epsilon$. (5.9)-(5.10) imply Theorem 2.3 for $\omega, \epsilon \rightarrow 0, \frac{\omega}{\epsilon} \rightarrow \infty$.

5.1.2 Part 2: $\omega, \epsilon \rightarrow 0, \frac{\omega}{\epsilon} \rightarrow \sigma \in[0, \infty)$.

For $v \in(0,1), \beta \in(0, \infty)$, and $i_{1}, i_{2} \in\{1, \cdots, n\}$, we find $\widetilde{\mathbb{X}}_{v, \beta}^{\left(i_{1}\right)}, \widetilde{\mathbb{Y}}_{v, \beta}^{\left(i_{1}, i_{2}\right)} \in H_{p e r}^{1}\left(\mathbb{R}^{n}\right)$ satisfying

$$
\begin{aligned}
& \left\{\begin{array}{l}
\nabla \cdot\left(\mathbb{E}_{v^{2}} \mathbf{K}\left(\nabla \widetilde{\mathbb{X}}_{\nu, \beta}^{\left(i_{1}\right)}+\left(\mathcal{X}_{Y_{f}}+\mathbb{W}_{\beta} \mathcal{X}_{Y_{m}}\right) \vec{e}_{i_{1}}\right)\right)=0 \quad \text { in } Y, \\
\int_{Y_{f}} \widetilde{\mathbb{X}}_{v, \beta}^{\left(i_{1}\right)}(y) \mathrm{d} y=0,
\end{array}\right. \\
& \left\{\begin{aligned}
\nabla & \cdot\left(\mathbb{E}_{v^{2}} \mathbf{K}\left(\nabla \widetilde{\mathbb{Y}}_{\nu, \beta}^{\left(i_{1}, i_{2}\right)}+\widetilde{\mathbb{X}}_{\nu, \beta}^{\left(i_{2}\right)} \vec{e}_{i_{1}}\right)\right) \\
\quad= & \frac{\widetilde{\mathcal{K}}_{v, \beta}^{\left(i_{1}, i_{2}\right)}}{\left|Y_{f}\right|} \mathcal{X}_{Y_{f}}-\mathbb{E}_{v^{2}} \mathbf{K}\left(\partial_{i_{1}} \widetilde{\mathbb{X}}_{\nu, \beta}^{\left(i_{2}\right)}+\left(\mathcal{X}_{Y_{f}}+\mathbb{W}_{\beta} \mathcal{X}_{Y_{m}}\right) \delta_{i_{1}, i_{2}}\right) \quad \text { in } Y, \\
\int_{Y_{f}} \widetilde{\mathbb{Y}}_{\nu, \beta}^{\left(i_{1}, i_{2}\right)}(y) \mathrm{d} y=0, &
\end{aligned}\right.
\end{aligned}
$$

where $\vec{e}_{i_{1}}, \delta_{i_{1}, i_{2}}$ are same as those in (5.1), $\mathbb{W}_{\beta}$ is from (2.7)-(2.8), and $\widetilde{\mathcal{K}}_{\nu, \beta}^{\left(i_{1}, i_{2}\right)}$ is defined as

$$
\widetilde{\mathcal{K}}_{\nu, \beta}^{\left(i_{1}, i_{2}\right)} \equiv \int_{Y} \mathbb{E}_{v^{2}} \mathbf{K}\left(\partial_{i_{1}} \widetilde{\mathbb{X}}_{\nu, \beta}^{\left(i_{2}\right)}+\left(\mathcal{X}_{Y_{f}}+\mathbb{W}_{\beta} \mathcal{X}_{Y_{m}}\right) \delta_{i_{1}, i_{2}}\right) \mathrm{d} y .
$$

$\widetilde{\mathbb{X}}_{\nu, \beta}^{\left(i_{1}\right)}, \widetilde{\mathbb{Y}}_{v, \beta}^{\left(i_{1}, i_{2}\right)}$ in (5.11)-(5.12) are uniquely solvable by Lax-Milgram Theorem [16].

Lemma 5.1 Under A1-A2 and A4, the solution $\mathbb{W}_{\beta}$ of (2.7)-(2.8) satisfies

$$
\begin{cases}\mathbb{W}_{\beta}(x) \in[0,1] & \text { for any } x \in Y_{m}, \beta \in(0, \infty), \\ \left\|\mathbb{E}_{\beta^{2}} \mathbb{W}_{\beta}\right\|_{W^{2, p}\left(Y_{f}\right) \cap W^{2, p}\left(Y_{m}\right)} \leq c & \text { for any } p \in(1, \infty), \beta \in(0, \infty), \\ \int_{Y_{m}} \mathbf{T} \mathbb{W}_{\beta} d y \leq c \sqrt{|\beta \ln \beta|} \quad \text { if } \beta \in\left(0, \beta^{*}\right),\end{cases}
$$

where $\beta^{*}$ is a constant depending on $\mathbf{K}, \mathbf{T}$ and diam $|\Omega|$, and $c$ is independent of $\beta$.

Proof Corollary 3.2 [16] implies (5.14) . Theorems 9.11, 9.15 [16], extension method in Theorem 7.25 [16], and (5.14) 1 imply (5.14) 2 . For any $x \in Y_{m}$, we set $\eta_{x} \equiv \min _{z \in \partial Y_{m}}|z-x|$ and $\xi_{x} \equiv \max _{z \in \partial Y_{m}}|z-x|$. Next we fix $t \in \mathbb{R}$ and $x \in Y_{m}$ as well as define $\varphi(y) \equiv$ $\exp \left(\left(|y-x|^{2}-\eta_{x}^{2}\right) t\right)$ for $y \in Y_{m}$. Then $\varphi$ satisfies

$$
\beta^{2} \nabla \cdot(\mathbf{K} \nabla \varphi)-\mathbf{T} \varphi=\beta^{2} \varphi\left(4 t^{2} \mathbf{K}|y-x|^{2}+2 t(y-x) \nabla \mathbf{K}+2 t \mathbf{K} n-\mathbf{T} / \beta^{2}\right) .
$$

We find that there are $\beta^{*}<1$ and $c^{*}>0$ (depending on $\mathbf{K}, \mathbf{T}, \operatorname{diam}|\Omega|$ but not $x \in Y_{m}$ ) such that if $\beta \in\left(0, \beta^{*}\right), t=c^{*} / \beta$, and $x \in Y_{m}$, then 


$$
\begin{cases}\beta^{2} \nabla \cdot(\mathbf{K} \nabla \varphi)-\mathbf{T} \varphi \leq 0 & \text { in } B_{\xi_{x}}(x) \cap Y_{m} \\ \varphi \geq 1 & \text { in } Y_{m} \backslash B_{\eta_{x}}(x) .\end{cases}
$$

Corollary 3.2 [16], (2.7), and (5.15) imply

$$
0 \leq \mathbb{W}_{\beta} \leq \varphi \quad \text { on } Y_{m}
$$

For any $v>0$, define $Y_{m}(v) \equiv\left\{y \in Y_{m}\left|\min _{z \in \partial Y_{m}}\right| z-y \mid \geq v\right\}$. By (5.16), it is easy to see that if $\beta \in\left(0, \beta^{*}\right)$, then, for any $x \in Y_{m}\left(\sqrt{2|\beta \ln \beta| / c^{*}}\right)$,

$$
0 \leq \mathbb{W}_{\beta} \leq \beta \quad \text { on } B_{\eta_{x} / 2}(x) .
$$

So if $\beta \in\left(0, \beta^{*}\right), \int_{Y_{m}} \mathbf{T} \mathbb{W}_{\beta} \mathrm{d} y \leq c \sqrt{|\beta \ln \beta|}$ by (5.14) 1 and (5.17).

By Lemma 3.3, Lemma 5.1, and energy method, there is a $\omega_{0} \in(0,1)$ such that, for any $v \in\left(0, \omega_{0}\right)$ and $\beta \in(0, \infty)$,

$$
\left\{\begin{array}{l}
\left\|\widetilde{\mathbb{X}}_{\nu, \beta}^{\left(i_{1}\right)}\right\|_{W^{1, p}\left(Y_{f}\right) \cap W^{1, p}\left(Y_{m}\right)},\left\|\widetilde{\mathbb{Y}}_{\nu, \beta}^{\left(i_{1}, i_{2}\right)}\right\|_{W^{2, p}\left(Y_{f}\right) \cap W^{2, p}\left(Y_{m}\right)} \leq c, \\
\left\|\widetilde{\mathbb{X}}_{\nu, \beta}^{\left(i_{1}\right)}, \widetilde{\mathbb{Y}}_{\nu, \beta}^{\left(i_{1}, i_{2}\right)}\right\|_{C^{2, \alpha}\left(\overline{Y_{f}}\right) \cap C^{2, \alpha}\left(\overline{Y_{m}}\right)} \leq \frac{c}{\beta^{2}}, \\
\left|\widetilde{\mathcal{K}}_{\nu, \beta}^{\left(i_{1}, i_{2}\right)}-\mathcal{K}_{0}^{\left(i_{1}, i_{2}\right)}\right| \leq c\left(\frac{v}{\beta}+v^{2}\right) \quad \text { as } v \rightarrow 0, \\
\left|\mathcal{T}_{\tau}-\mathcal{T}_{\sigma}\right| \leq \begin{cases}c\left|\frac{\tau^{2}}{\sigma^{2}}-1\right| & \text { if } \tau \rightarrow \sigma \in(0, \infty), \\
c \sqrt{|\tau \ln \tau|} & \text { if } \tau \rightarrow \sigma=0,\end{cases}
\end{array}\right.
$$

where $p \in(n, \infty), \alpha \in(0,1), \mathcal{K}_{0}^{\left(i_{1}, i_{2}\right)}$ is the $\left(i_{1}, i_{2}\right)$ th component of $\mathcal{K}_{0}$ (see (2.9)), and $c$ is a constant independent of $\nu, \beta, \tau, \sigma$. See (5.13) for $\widetilde{\mathcal{K}}_{\nu, \beta}^{\left(i_{1}, i_{2}\right)}$, and see (2.11) for $\mathcal{T}_{\sigma}$. Define $\widetilde{\mathbb{X}}_{\omega, \beta, \epsilon}(x) \equiv \epsilon \widetilde{\mathbb{X}}_{\omega, \beta}\left(\frac{x}{\epsilon}\right) \widetilde{\mathbb{Y}}_{\omega, \beta} \equiv\left(\widetilde{\mathbb{Y}}_{\omega, \beta}^{\left(i_{1}, i_{2}\right)}\right)$ and $\widetilde{\mathbb{Y}}_{\omega, \beta, \epsilon}(x) \equiv \epsilon^{2} \widetilde{\mathbb{Y}}_{\omega, \beta}\left(\frac{x}{\epsilon}\right)$ for $\beta \in(0, \infty), \omega, \epsilon \in(0,1), i_{1}, i_{2} \in \mathbb{Z}$.

Let $\Psi_{\omega, \epsilon}$ be the solution of (1.1) with $V_{\omega, \epsilon}=0, \Psi$ be the solution of (2.12), and

$$
\Phi_{\omega, \epsilon} \equiv \Psi_{\omega, \epsilon}-\left(\mathcal{X}_{\Omega_{f}^{\epsilon}}+\mathbb{W}_{\frac{\omega}{\epsilon}, \epsilon, 0} \mathcal{X}_{\Omega_{m}^{\epsilon}}\right) \Psi-\mathbb{W}_{\frac{\omega}{\epsilon}, \epsilon, 2} \Psi \mathcal{X}_{\Omega_{f}^{\epsilon}}-\widetilde{\mathbb{X}}_{\omega, \frac{\omega}{\epsilon}, \epsilon} \nabla \Psi-\widetilde{\mathbb{Y}}_{\omega, \frac{\omega}{\epsilon}, \epsilon} \nabla^{2} \Psi
$$

in $\Omega$. See remark after (2.8) for $\mathbb{W}_{\frac{\omega}{\epsilon}, \epsilon, i}$. By (2.7)-(2.8), (5.11)-(5.12), (5.18), and Lemma 5.1 with $\beta=\frac{\omega}{\epsilon}$, we obtain

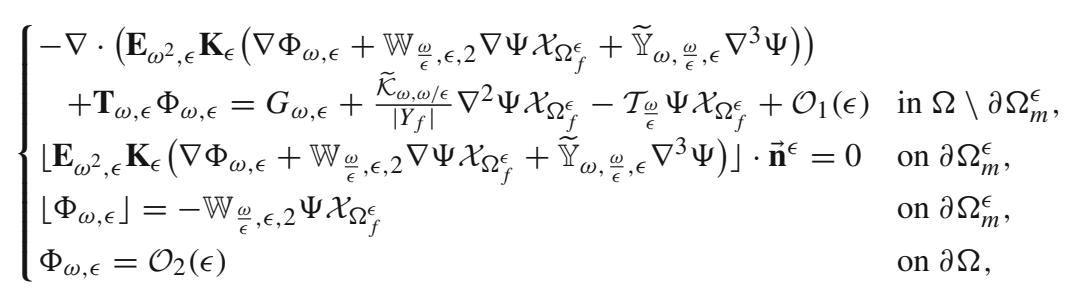

where $\mathcal{O}_{1}(v), \mathcal{O}_{2}(v)$ are same as those in Part 1. See (2.2) for (5.19) 2,3 . Let us define

$$
\varphi_{\omega, \epsilon} \equiv \begin{cases}\Phi_{\omega, \epsilon}+\mathbb{W}_{\frac{\omega}{\epsilon}, \epsilon, 2} \Psi \mathcal{X}_{\Omega_{f}^{\epsilon}} & \text { on } \Omega_{f}^{\epsilon} \\ \Phi_{\omega, \epsilon} & \text { on } \Omega_{m}^{\epsilon}\end{cases}
$$


By (2.12)-(2.13), (5.18), and Lemma 5.1, $\varphi_{\omega, \epsilon}$ satisfies

$$
\begin{cases}-\nabla \cdot\left(\mathbf{E}_{\omega^{2}, \epsilon} \mathbf{K}_{\epsilon}\left(\nabla \varphi_{\omega, \epsilon}+\mathcal{O}_{1}(\epsilon)\right)\right)+\mathbf{T}_{\omega, \epsilon} \varphi_{\omega, \epsilon}=G_{\omega, \epsilon}-\mathcal{G} \mathcal{X}_{\Omega_{f}^{\epsilon}}+\tilde{\mathcal{X}} & \text { in } \Omega, \\ \varphi_{\omega, \epsilon}=\mathcal{O}_{2}(\epsilon) & \text { on } \partial \Omega,\end{cases}
$$

where

$$
\tilde{\mathcal{X}} \equiv \begin{cases}\mathcal{O}_{1}\left(\epsilon+\left|\frac{\omega^{2}}{\sigma^{2} \epsilon^{2}}-1\right|\right) & \text { if } \frac{\omega}{\epsilon} \rightarrow \sigma \in(0, \infty), \\ \mathcal{O}_{1}\left(\epsilon+\left|\frac{\omega}{\epsilon} \ln \frac{\omega}{\epsilon}\right|^{\frac{1}{2}}\right) & \text { if } \frac{\omega}{\epsilon} \rightarrow 0\end{cases}
$$

We write the $\varphi_{\omega, \epsilon}$ as $\varphi_{\omega, \epsilon}=\check{\varphi}_{\omega, \epsilon}+\widehat{\varphi}_{\omega, \epsilon}$, where $\check{\varphi}_{\omega, \epsilon}$ satisfies

$$
\begin{cases}-\nabla \cdot\left(\mathbf{E}_{\omega^{2}, \epsilon} \mathbf{K}_{\epsilon} \nabla \check{\varphi}_{\omega, \epsilon}\right)+\mathbf{T}_{\omega, \epsilon} \check{\varphi}_{\omega, \epsilon}=0 & \text { in } \Omega \\ \check{\varphi}_{\omega, \epsilon}=\mathcal{O}_{2}(\epsilon) & \text { on } \partial \Omega,\end{cases}
$$

and $\widehat{\varphi}_{\omega, \epsilon}$ satisfies

$$
\begin{cases}-\nabla \cdot\left(\mathbf{E}_{\omega^{2}, \epsilon} \mathbf{K}_{\epsilon}\left(\nabla \widehat{\varphi}_{\omega, \epsilon}+\mathcal{O}_{1}(\epsilon)\right)\right)+\mathbf{T}_{\omega, \epsilon} \widehat{\varphi}_{\omega, \epsilon}=G_{\omega, \epsilon}-\mathcal{G} \mathcal{X}_{\Omega_{f}^{\epsilon}}+\tilde{\mathcal{X}} & \text { in } \Omega, \\ \widehat{\varphi}_{\omega, \epsilon}=0 & \text { on } \partial \Omega .\end{cases}
$$

By Theorem 8.1 [16], the solution of (5.20) satisfies

$$
\left\|\check{\varphi}_{\omega, \epsilon}\right\|_{L^{\infty}(\Omega)}=\left\|\check{\varphi}_{\omega, \epsilon}\right\|_{L^{\infty}(\partial \Omega)} \leq c \epsilon,
$$

where $c$ is independent of $\omega, \epsilon$. Next we consider (5.21) for $\frac{\omega}{\epsilon} \rightarrow \sigma \in(0, \infty)$ and $\frac{\omega}{\epsilon} \rightarrow 0$ separately.

Case 1: $\frac{\omega}{\epsilon} \rightarrow \sigma \in(0, \infty)$. By Theorem 2.1 and A6, the solution of (5.21) satisfies

$$
\left\|\mathbf{E}_{\omega, \epsilon} \widehat{\varphi}_{\omega, \epsilon}\right\|_{L^{\infty}(\Omega)} \leq c\left(\max \left\{\omega, \epsilon,\left|\omega^{2} /(\sigma \epsilon)^{2}-1\right|\right\}+\left\|G_{\omega, \epsilon}-\mathcal{G}\right\|_{L^{n+\delta}\left(\Omega_{f}^{\epsilon}\right)},\right.
$$

where $c$ is independent of $\omega, \epsilon$. Employing (5.22)-(5.23) and modifying the argument of Part 1 , we obtain Theorem 2.3 for $\omega, \epsilon \rightarrow 0, \frac{\omega}{\epsilon} \rightarrow \sigma \in(0, \infty)$ case.

Case 2: $\frac{\omega}{\epsilon} \rightarrow 0$. If $\widehat{\varphi}_{\omega, \epsilon}$ is the solution of (5.21), we multiply (5.21) by $\left|\widehat{\varphi}_{\omega, \epsilon}\right|^{n+\delta-2} \widehat{\varphi}_{\omega, \epsilon}$ and integrate by part to see, by A6,

$$
\left\|\widehat{\varphi}_{\omega, \epsilon}\right\|_{L^{n+\delta}(\Omega)} \leq c\left(\max \left\{\omega, \epsilon,\left|\frac{\omega}{\epsilon} \ln \frac{\omega}{\epsilon}\right|^{\frac{1}{2}}\right\}+\left\|G_{\omega, \epsilon}-\mathcal{G}\right\|_{L^{n+\delta}\left(\Omega_{f}^{\epsilon}\right)}\right),
$$

where $c$ is independent of $\omega, \epsilon$. By (5.22) and (5.24), we obtain Theorem 2.3 for $\omega, \epsilon \rightarrow$ $0, \frac{\omega}{\epsilon} \rightarrow 0$ case.

\subsection{Proof of Theorem 2.4}

The proof is similar to that of Theorem 2.3. Let us assume that A1-A3, A4', A6, and A7 hold. For each $v \in(0,1)$, we find $\breve{\mathbb{W}}_{v} \in H_{\text {per }}^{1}\left(\mathbb{R}^{n}\right)$ satisfying

$$
\left\{\begin{array}{l}
\nabla \cdot\left(\mathbb{E}_{v^{2}} \mathbf{K} \nabla \breve{\mathbb{W}}_{\nu}\right)=\left(\mathbb{E}_{\nu} \mathbf{P}-\breve{\mathcal{T}}\right) \mathcal{X}_{Y_{f}} \quad \text { in } Y, \\
\int_{Y_{f}} \breve{\mathbb{W}}_{\nu}(y) \mathrm{d} y=0
\end{array}\right.
$$

See A7 for $\mathbf{P}$, and (2.15) for constant $\breve{\mathcal{T}}$. By Lax-Milgram Theorem [16], Lemma 3.3, and A7, $\breve{\mathbb{W}}_{v}$ is uniquely solvable and

$$
\left\|\breve{\mathbb{W}}_{\nu}\right\|_{C^{2}\left(\overline{Y_{f}}\right) \cap C^{2}\left(\overline{Y_{m}}\right)} \leq c,
$$


where $c$ is a constant independent of $v$. Define $\breve{\mathbb{W}}_{\nu, s}(x) \equiv s^{2} \breve{\mathbb{W}}_{\nu}\left(\frac{x}{s}\right)$ for $v, s \in(0,1)$.

Let $\Psi_{\omega, \epsilon}$ be the solution of (1.1) with $V_{\omega, \epsilon}=0, \Psi$ be the solution of (2.16), and

$$
\varphi_{\omega, \epsilon} \equiv \Psi_{\omega, \epsilon}-\Psi-\breve{\mathbb{W}}_{\omega, \epsilon} \Psi-\mathbb{X}_{\omega, \epsilon} \nabla \Psi-\mathbb{Y}_{\omega, \epsilon} \nabla^{2} \Psi \quad \text { in } \Omega .
$$

See (2.5) for $\mathbb{X}_{\omega, \epsilon}$ and (5.1) for $\mathbb{Y}_{\omega, \epsilon}$. By (2.16), (3.17), (3.19), (5.1)-(5.2), and (5.25)-(5.26), we obtain

$$
\left\{\begin{array}{rlrl}
-\nabla & \cdot\left(\mathbf{E}_{\omega^{2}, \epsilon} \mathbf{K}_{\epsilon}\left(\nabla \varphi_{\omega, \epsilon}+\mathcal{O}_{1}\left(\epsilon^{2}\right)\right)\right)+\mathbf{T}_{\omega, \epsilon} \varphi_{\omega, \epsilon} & \\
& =\mathcal{O}_{1}(\epsilon+\omega) \mathcal{X}_{\Omega_{f}^{\epsilon}}+\mathcal{O}_{1}(\omega) \mathcal{X}_{\Omega_{m}^{\epsilon}}+\left(G_{\omega, \epsilon}-\mathcal{G}\right) \mathcal{X}_{\Omega_{f}^{\epsilon}} & & \text { in } \Omega, \\
\varphi_{\omega, \epsilon} & =\mathcal{O}_{2}(\epsilon) & & \text { on } \partial \Omega,
\end{array}\right.
$$

where $\mathcal{O}_{1}(v), \mathcal{O}_{2}(v)$ are defined as those in Part 1. See (2.15) for $\mathcal{G}$. Modifying the argument of Part 1 in Sect. 5.1 and employing Theorem 2.2, we obtain Theorem 2.4.

\subsection{Proof of Theorem 2.5}

We assume A1-A3 and A6. Let $\Psi_{\omega, \epsilon}$ be the solution of (1.1) with $V_{\omega, \epsilon}=\mathbf{T}_{\omega, \epsilon}=0, \Psi$ be the solution of (2.18), and define

$$
\varphi_{\omega, \epsilon} \equiv \Psi_{\omega, \epsilon}-\Psi-\mathbb{X}_{\omega, \epsilon} \nabla \Psi-\mathbb{Y}_{\omega, \epsilon} \nabla^{2} \Psi \quad \text { in } \Omega .
$$

See (2.5) for $\mathbb{X}_{\omega, \epsilon}$ and (5.1) for $\mathbb{Y}_{\omega, \epsilon}$. By (2.18), (3.17), (3.19), and (5.2), $\varphi_{\omega, \epsilon}$ satisfies

$$
\left\{\begin{aligned}
-\nabla & \cdot\left(\mathbf{E}_{\omega^{2}, \epsilon} \mathbf{K}_{\epsilon}\left(\nabla \varphi_{\omega, \epsilon}+\mathcal{O}_{1}\left(\epsilon^{2}\right)\right)\right) & & \\
& =\mathcal{O}_{1}(\epsilon+\omega) \mathcal{X}_{\Omega_{f}^{\epsilon}}+\mathcal{O}_{1}(\omega) \mathcal{X}_{\Omega_{m}^{\epsilon}}+\left(G_{\omega, \epsilon}-\mathcal{G}\right) \mathcal{X}_{\Omega_{f}^{\epsilon}} & & \text { in } \Omega, \\
\varphi_{\omega, \epsilon} & =\mathcal{O}_{2}(\epsilon) & & \text { on } \partial \Omega .
\end{aligned}\right.
$$

Modifying the argument of Part 1 in Sect. 5.1 and employing Theorem 2.2, we obtain Theorem 2.5 .

Acknowledgments The author would like to thank the anonymous referee's valuable suggestions for improving the presentation of this paper. This research is supported by the Grant number NSC 101-2115-M-009-012 from the research program of National Science Council of Taiwan.

\section{References}

1. Acerbi, E., Chiado Piat, V., Dal Maso, G., Percivale, D.: An extension theorem from connected sets, and homogenization in general periodic domains. Nonlinear Anal. 18, 481-496 (1992)

2. Adams, R.A.: Sobolev Spaces. Academic Press, Massachusetts (2003)

3. Allaire, G.: Homogenization and two-scale convergence. SIAM I. Math. Anal. 23, 1482-1518 (1992)

4. Avellaneda, M., Lin, F.-H.: Homogenization of elliptic problems with $L^{p}$ boundary data. Appl. Math. Opt. 15, 93-107 (1987)

5. Avellaneda, M., Lin, F.-H.: Compactness methods in the theory of homogenization. Commun. Pure Appl. Math. XI, 803-847 (1987)

6. Babych, N.O., Kamotski, I.V., Smyshlyaev, V.P.: Homogenization of spectral problems in bounded domains with doubly high contrasts. Netw. Heterog. Media 3(3), 413-436 (2008)

7. Bakhvalov, N., Panasenko, G.: Homogenisation: Averaging Processes in Periodic Media: Mathematical Problems in the Mechanics of Composite Materials. Kluwer Academic Publishers, Heidelberg (1989)

8. Bellieud, M., Bouchitt, G.: Homogenization of elliptic problems in a fiber reinforced structure. Ann. Scuola Norm. Sup. Pisa Cl. Sci. (4) 26(3), 407-436 (1998)

9. Bellieud, M., Bouchitt, G.: Homogenization of degenerate elliptic equations. C. R. Acad. Sci. Paris Sr. I Math. 327(8), 787-792 (1998) 
10. Berkowitz, B., et al.: Physical pictures of transport in heterogeneous media: advection-dispersion, random walk, and fractional derivative formulations. Water Resour. Res. 38(10), 1191-1194 (2002)

11. Briane, M., Capdeboscq, Y., Nguyen, L.: Interior regularity estimates in high conductivity homogenization and application. Arch. Ration. Mech. Anal. 207(1), 75-137 (2013)

12. Briane, M., Tchou, N.: Fibered microstructures for some nonlocal Dirichlet forms. Ann. Scuola Norm. Sup. Pisa Cl. Sci. (4) 30(3-4), 681-711 (2001)

13. Cafarelli, L.A., Peral, I.: On $W^{1, p}$ estimates for elliptic equations in divergence form. Commun. Pure Appl. Math. 51, 1-21 (1998)

14. Chen, G., Zhou, J.: Boundary Element Methods. Academic Press, Massachusetts (1992)

15. Giaquinta, M.: Multiple Integrals in the Calculus of Variations, (Study 105, Annals of Math. Studies). Princeton Univ. Press, Princeton (1983)

16. Gilbarg, D., Trudinger, N.S.: Elliptic Partial Differential Equations of Second Order, 2nd edn. Springer, Berlin (1983)

17. Jikov, V.V., Kozlov, S.M., Oleinik, O.A.: Homogenization of Differential Operators and Integral Functions. Springer, Berlin (1994)

18. Klein, V., Peszynska, M.: Adaptive double-diffusion model and comparison to a highly heterogeneous micro-model. J. Appl. Math. 2012, 1-26, Art. ID 938727 (2012). doi:10.1155/2012/938727

19. Ladyzhenskaya, O.A., Ural'tseva, N.N.: Elliptic and Quasilinear Elliptic Equations. Academic Press, Massachusetts (1968)

20. Li, Y.Y., Vogelius, M.: Gradient estimates for solutions to divergence form elliptic equations with discontinuous coefficients. Arch. Ration. Mech. Anal. 153, 91-151 (2000)

21. Maugeri, A., Palagachev, D., Lubomira, G.: Softova, elliptic and parabolic equations with discontinuous coefficients. Wiley-VCH, Berlin (2000)

22. Meyers, N.G.: An $L^{p}$-estimate for the gradient of solutions of second order elliptic divergence equations. Ann. Scuola Norm. Sup. Pisa (3) 17, 189-206 (1963)

23. Oleinik, O.A., Shamaev, A.S., Tosifan, G.A.: Mathematical Problems in Elasticity and Homogenization. North-Holland, Amsterdam (1992)

24. Schweizer, B.: Uniform estimates in two periodic homogenization problems. Commun. Pure Appl. Math. LIII, 1153-1176 (2000)

25. Shen, Z.: $W^{1, p}$ estimates for elliptic homogenization problems in nonsmooth domain. Indiana Univ. Math. J. 57(5), 2283-2298 (2008)

26. Sili, A.: Homogenization of a nonlinear monotone problem in an anisotropic medium. Math. Models Methods Appl. Sci. 14(3), 329-353 (2004)

27. Yeh, L.-M.: Holder estimate for non-uniform parabolic equations in highly heterogeneous media. Nonlinear Anal. 75, 3723-3745 (2012) 